\title{
L'onde F dans tous ses états
}

CLINICAL MOTOR ELECTRONEUROGRAPHY:

Evoked Responses Beyond the M-Wave

Ectopic Activity

An Atlas with 400 Figures

By

Gérard Roth

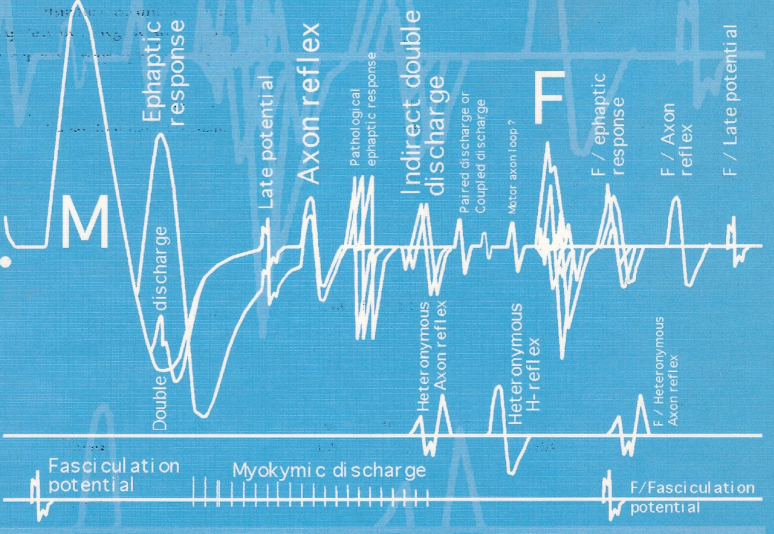

ELSEVIER

\author{
F. Wang \\ N. Massart \\ J.F. Kaux \\ O. Bouquiaux
}




\section{État civil de l’onde F}

\section{Origine}

\section{Caractéristiques}




\section{Origine et conditions d'obtention}

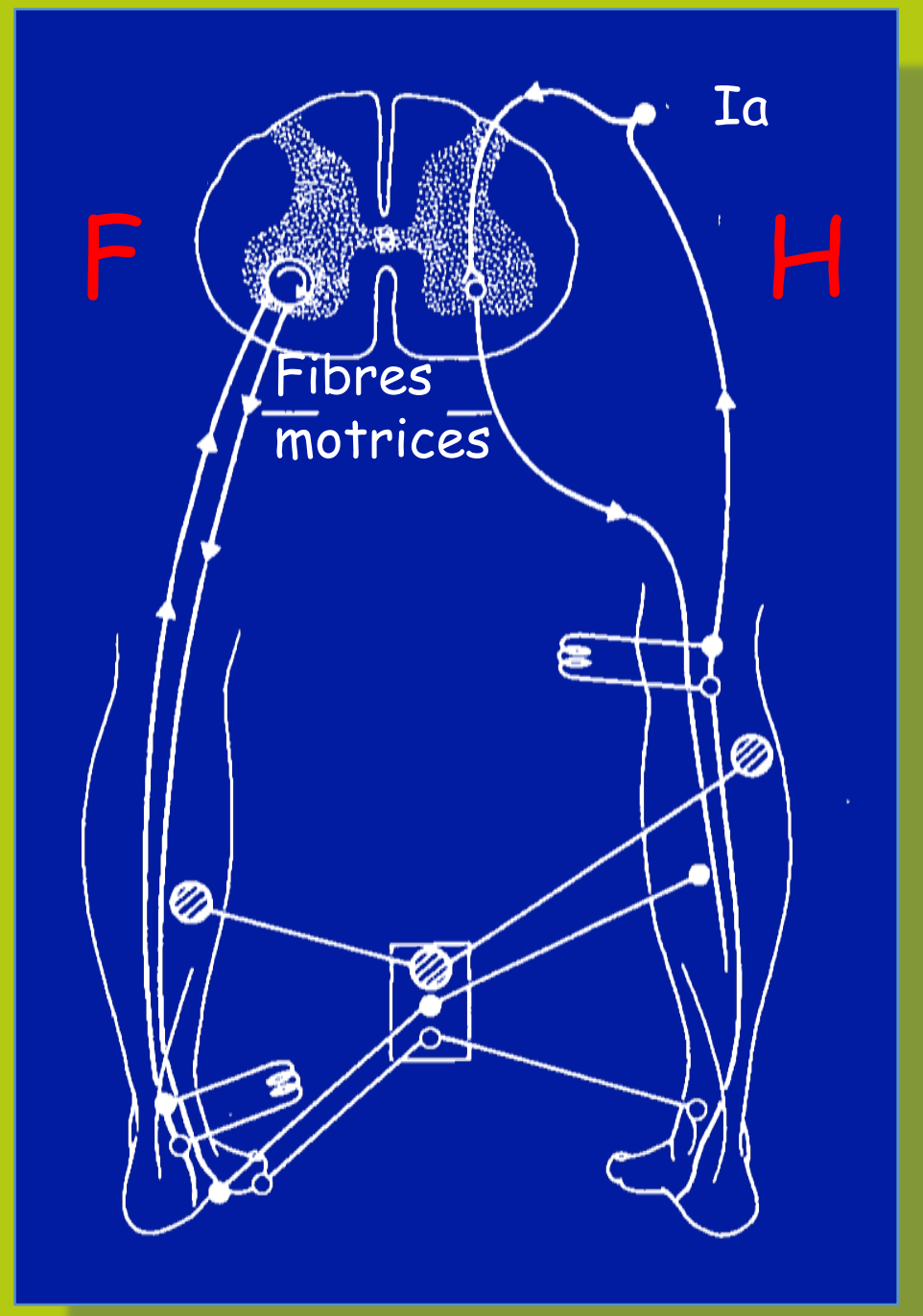

(1) Magladery et McDougal (1950): réponse $\mathrm{F}$ chez l'homme, au niveau du pied (F pour foot)

(2) Dawson et Merton (1956): réponse $\mathrm{F}$ ne $>$ pas d'un réflexe

(3) afférence $=$ efférence $=$ axone $\alpha$

(4) réponses $\mathrm{F}=$ décharges des motoneurones spinaux activés de façon rétrograde par les volées d' influx centripètes 


\section{Caractéristiques}

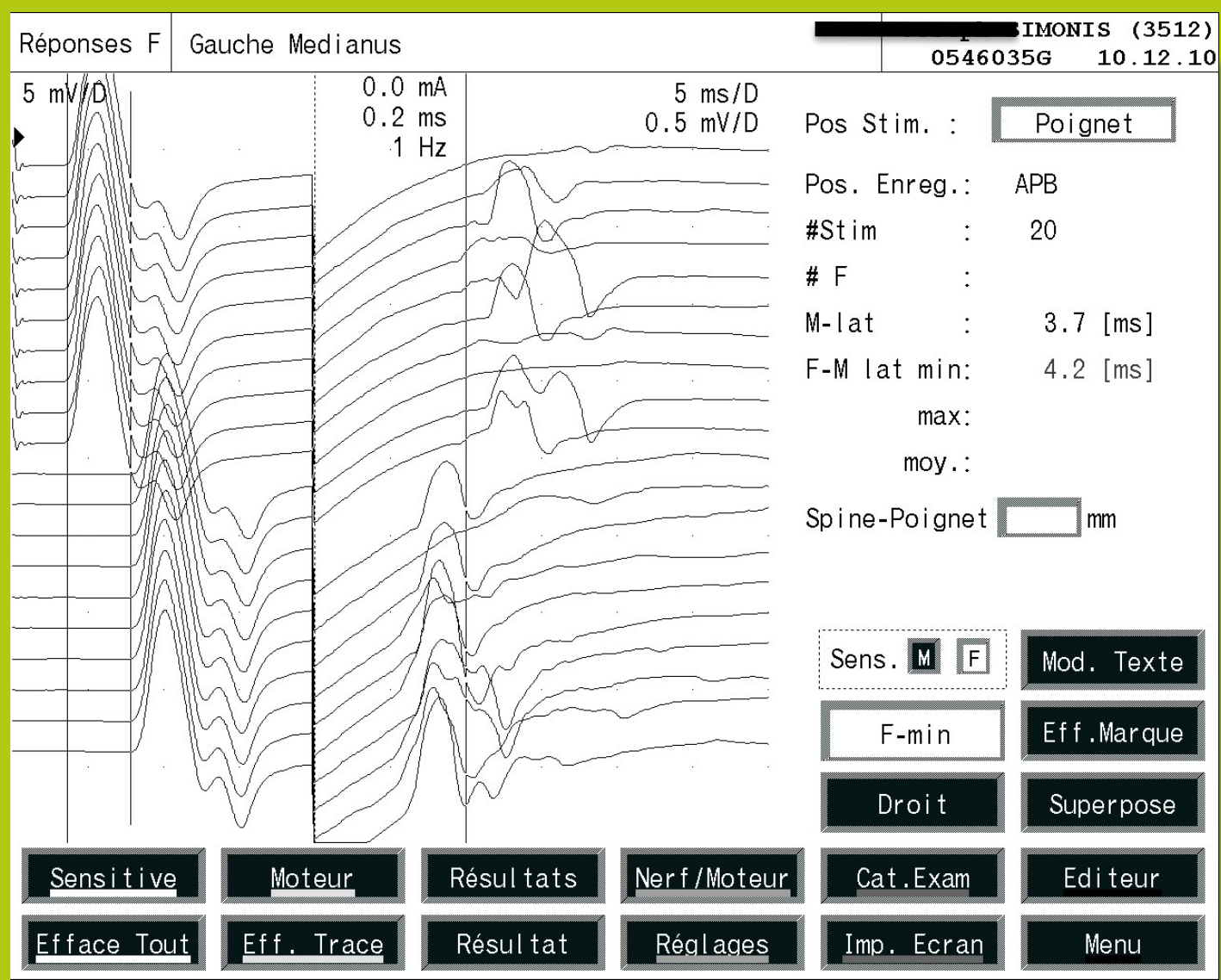

(1) non-physiologique

(2) spinale

(3) tardive

(4) indirecte

(5) variable :

- latence

- amplitude

- durée

-forme 


\section{Caractéristiques}

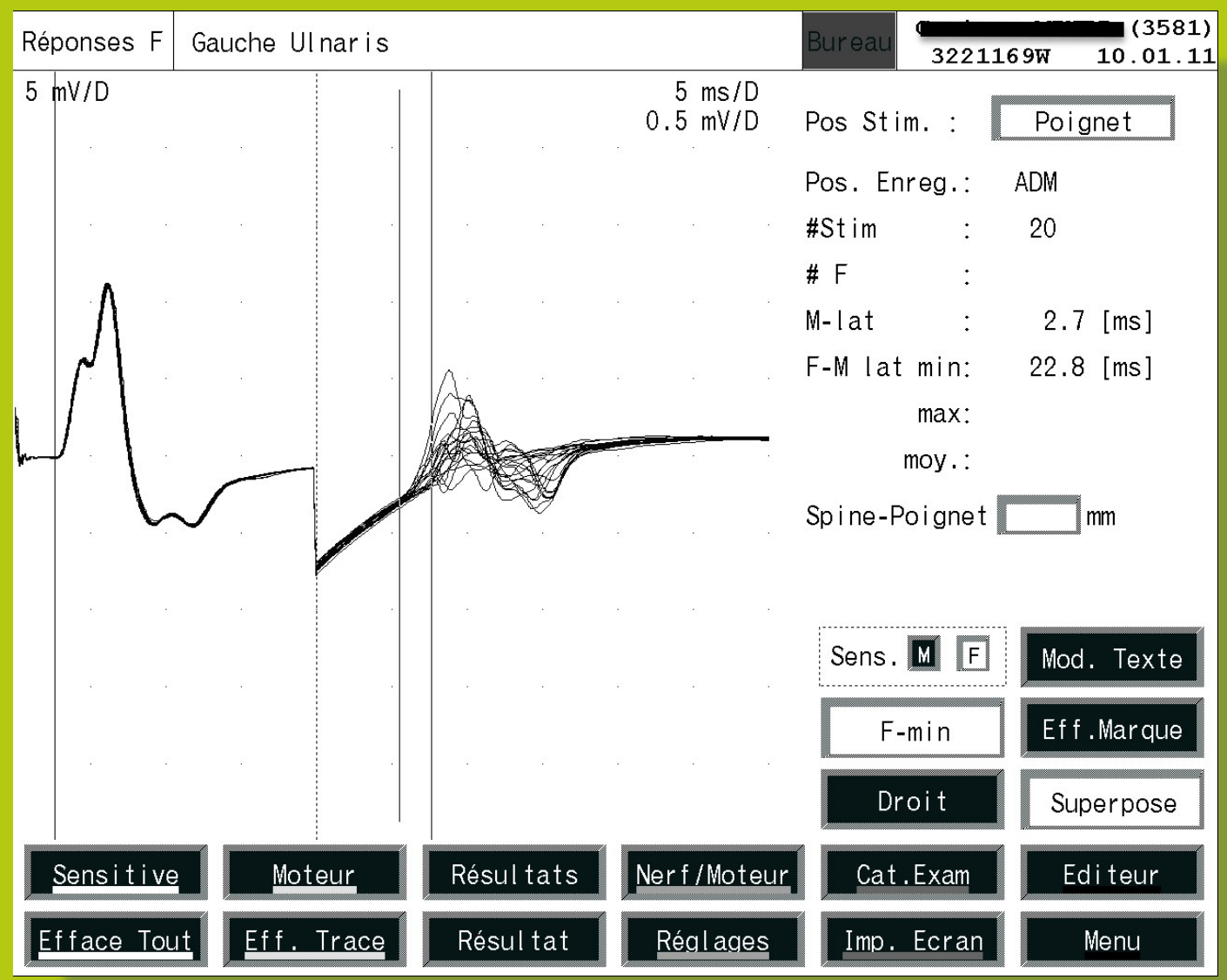

(1) non-physiologique

(2) spinale

(3) tardive

(4) indirecte

(5) variable :

- latence

- amplitude

- durée

-forme 


\title{
États de grâce
}

\author{
L’onde F unitaire
}




\section{L’onde F unitaire}

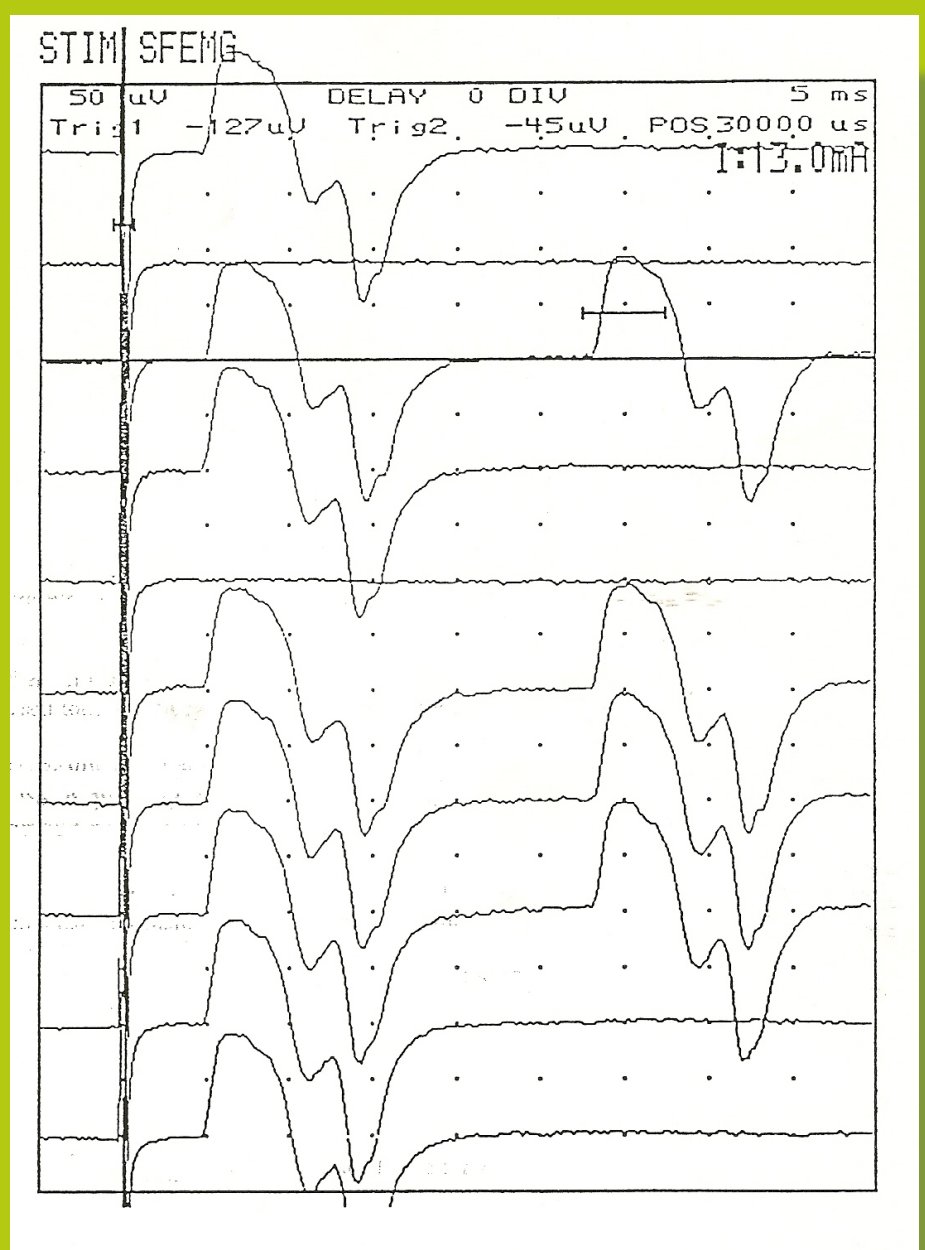

Enregistrement de surface

(1) un seul motoneurone spinal une seule unité motrice

(2) un seul axone moteur $\Rightarrow>$ pas de désynchronisation $=>$ les réponses précoce et tardive : morphologie identique

(3) stimulation nerveuse liminaire $=>$ réponse précoce inconstante

(4) réponse tardive inconstante latence stable

(5) pas de réponse tardive sans réponse précoce 


\section{L'onde F unitaire vs la réponse $\mathrm{H}$ unitaire}
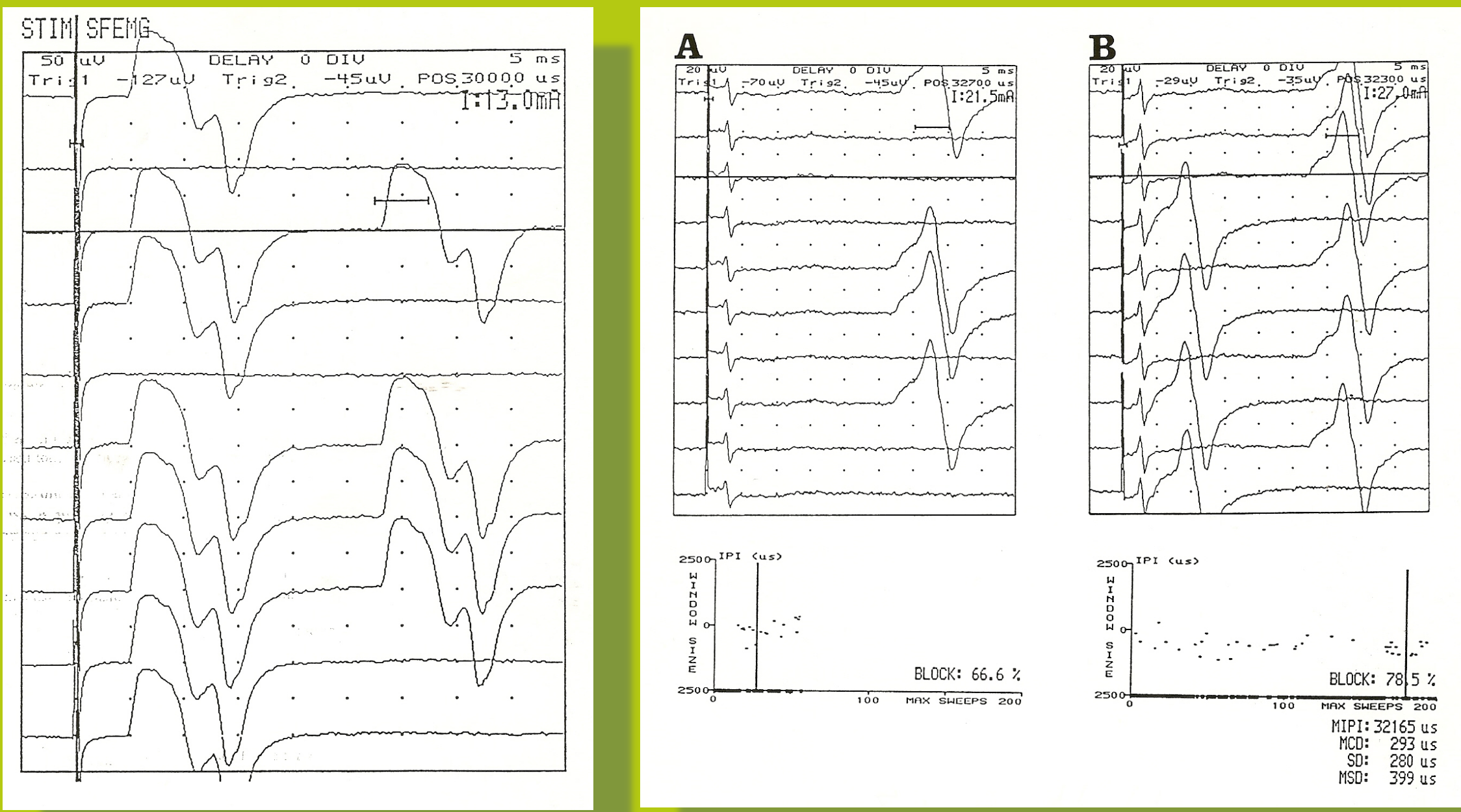


\section{États de confusion}

Réflexe H ou HH

Réflexe d'axone moteur

Décharge double indirecte

Réflexe tardif 


\section{Directes ou indirectes}

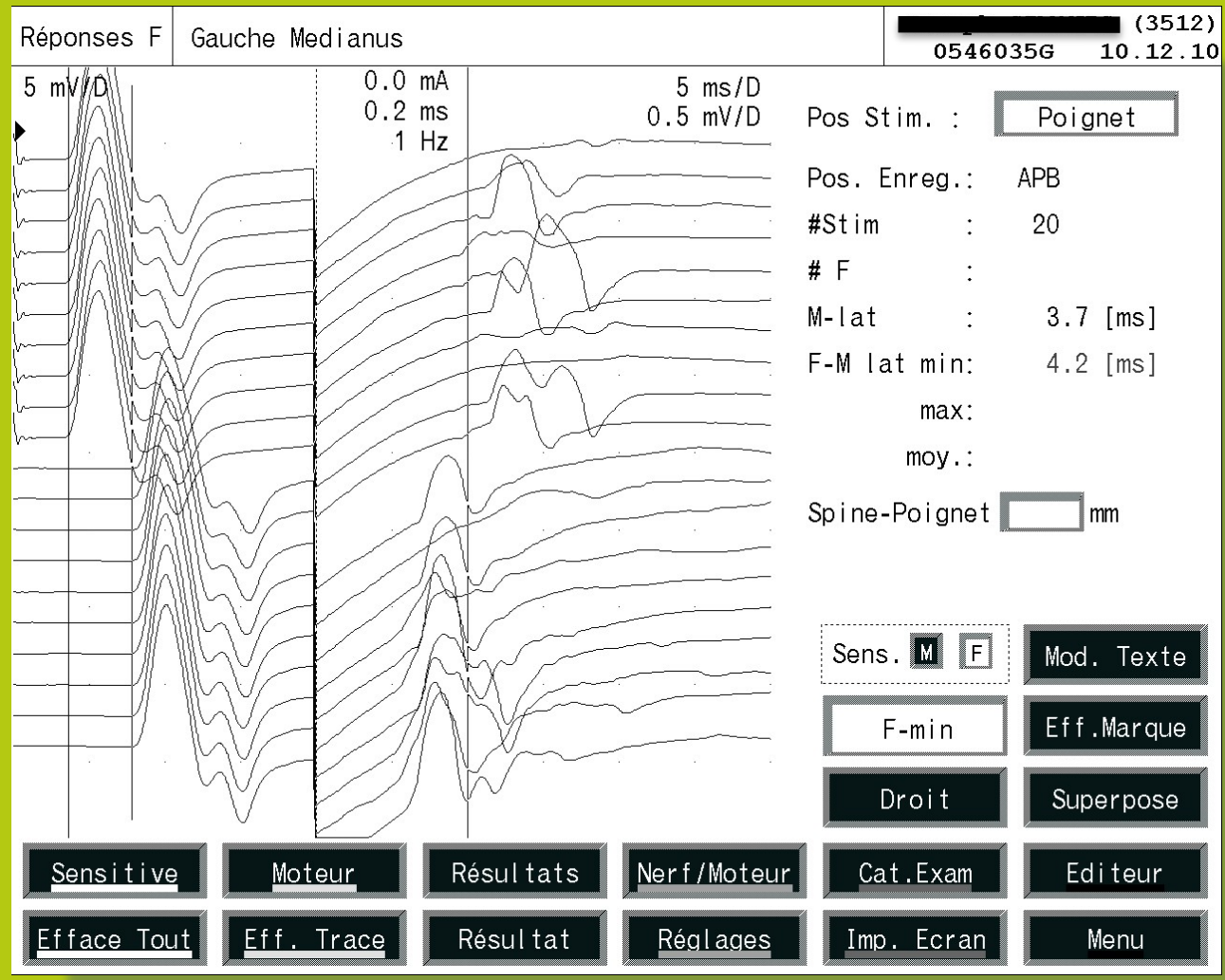

Directes

(1) potentiel tardif

(2) réponse éphaptique

(3) décharge double directe

Indirectes
(1) F et $\mathbf{H}$
(2) réflexe d'axone moteur
(3) décharge double indirecte 


\section{Réflexe d'axone moteur (RAM)}

(1) axonopathie chronique

(2) réinnervation terminale $=>$

(3) bifurcation axonale anormale

(4) une seule branche activée $\Rightarrow>$

RAM

(5) les deux branches activées

(stimulation plus intense) $\Rightarrow$

disparition de la réponse indirecte ("saut du RAM »)

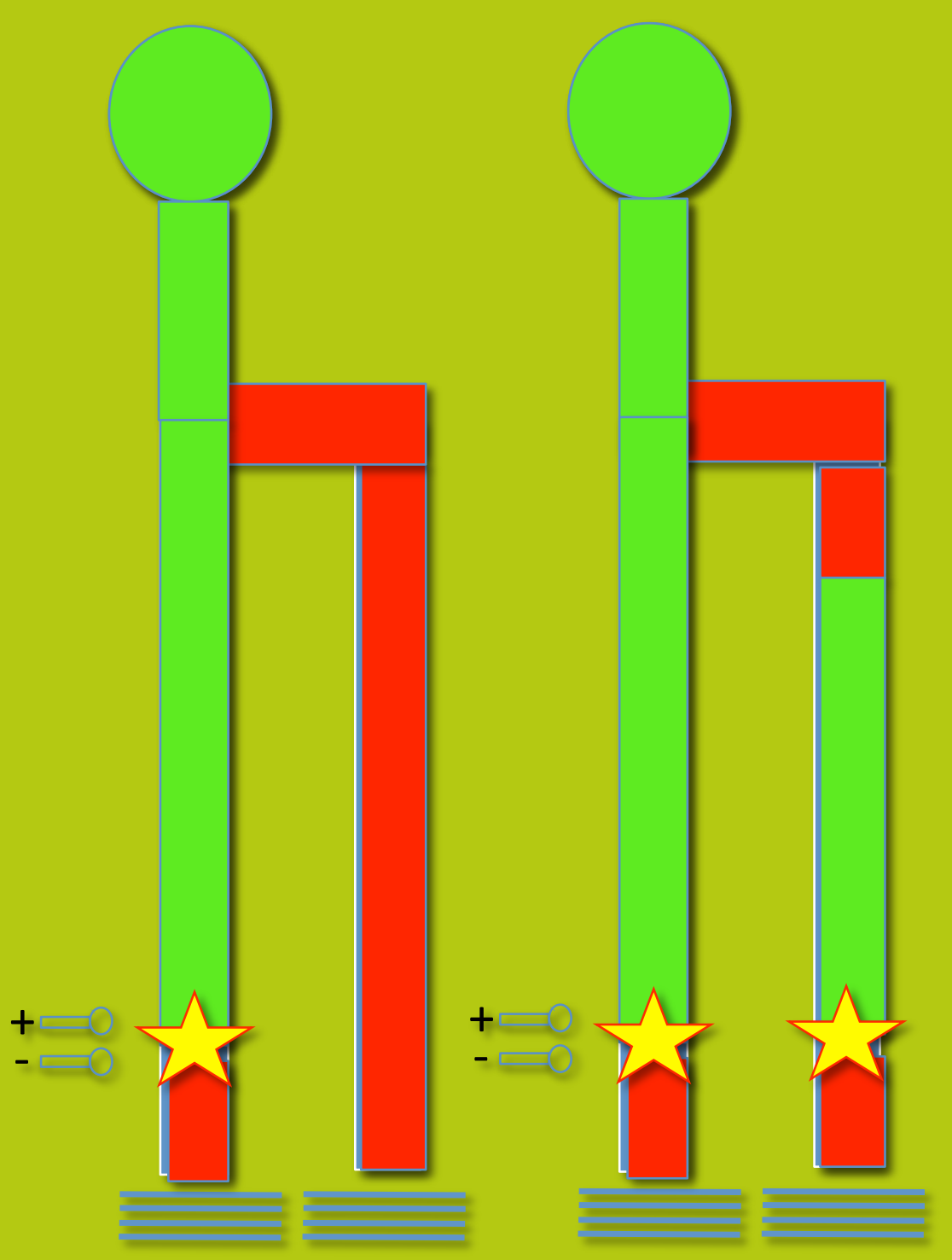




\section{Décharge double indirecte (DDI)}

(1) atteinte myélinique récente

(2) $=>$ foyers ectopiques de réactivation axonale proximale par des influx antidromiques

(3) $\Rightarrow>$ réponse intermédiaire

$$
=\mathrm{DDI}
$$

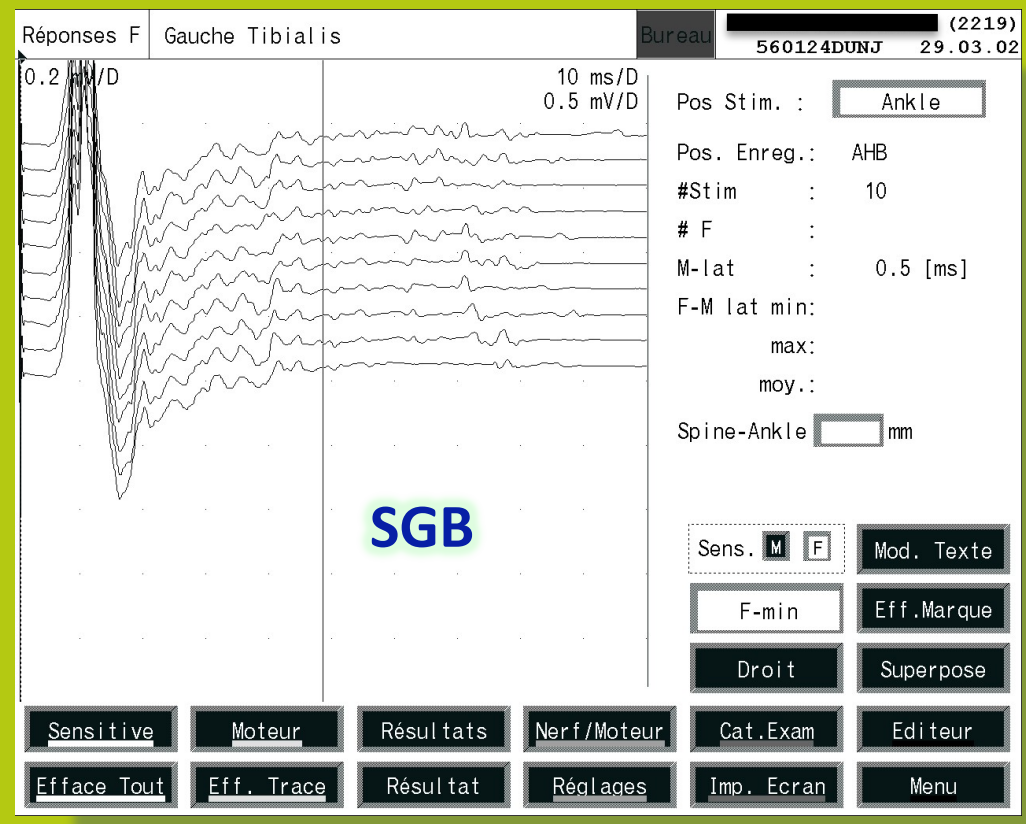




\section{Stimulation supramaximale : H}
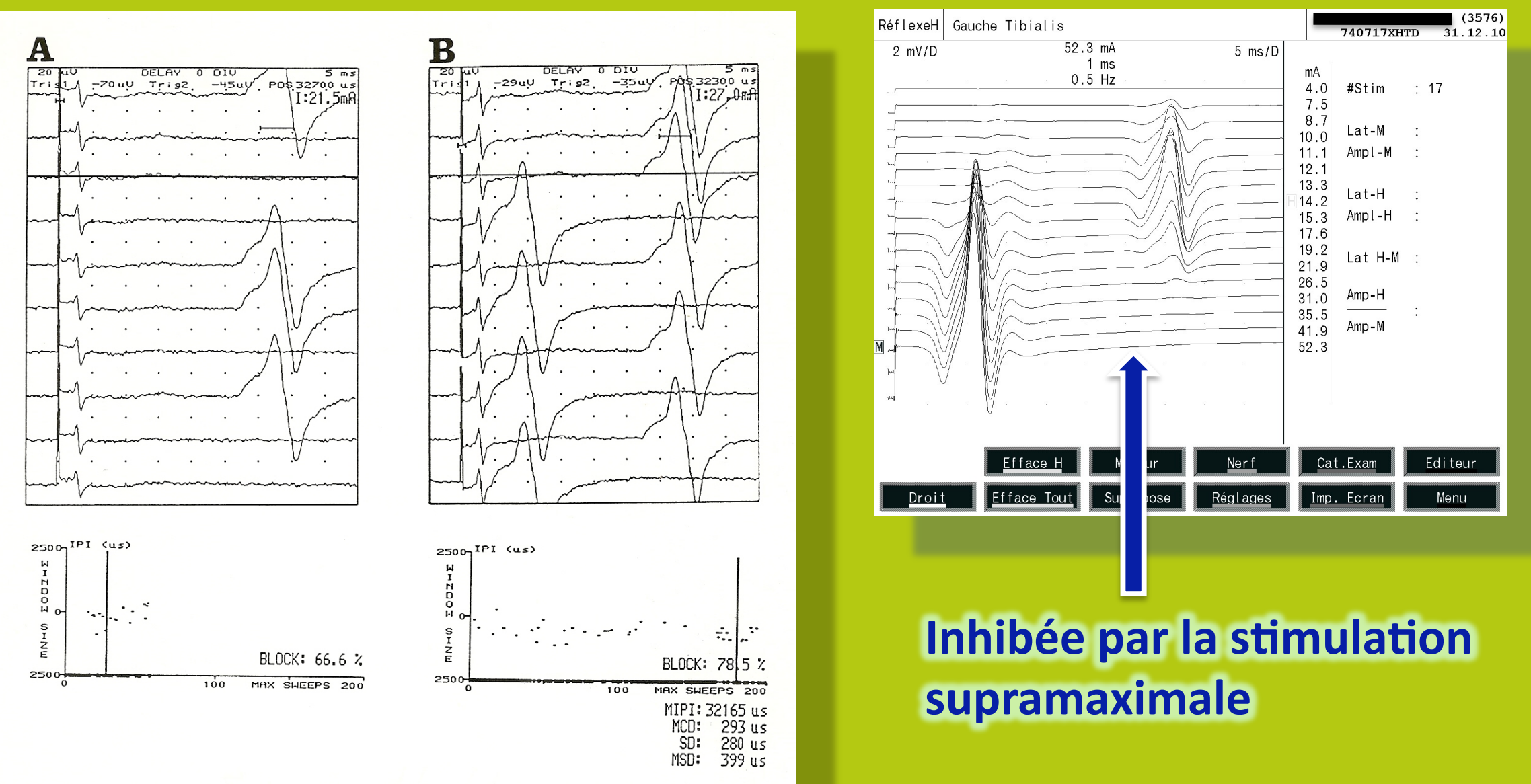

Inhibée par la stimulation supramaximale 


\section{F et $\mathrm{H}$ : chocs sous-maximaux}

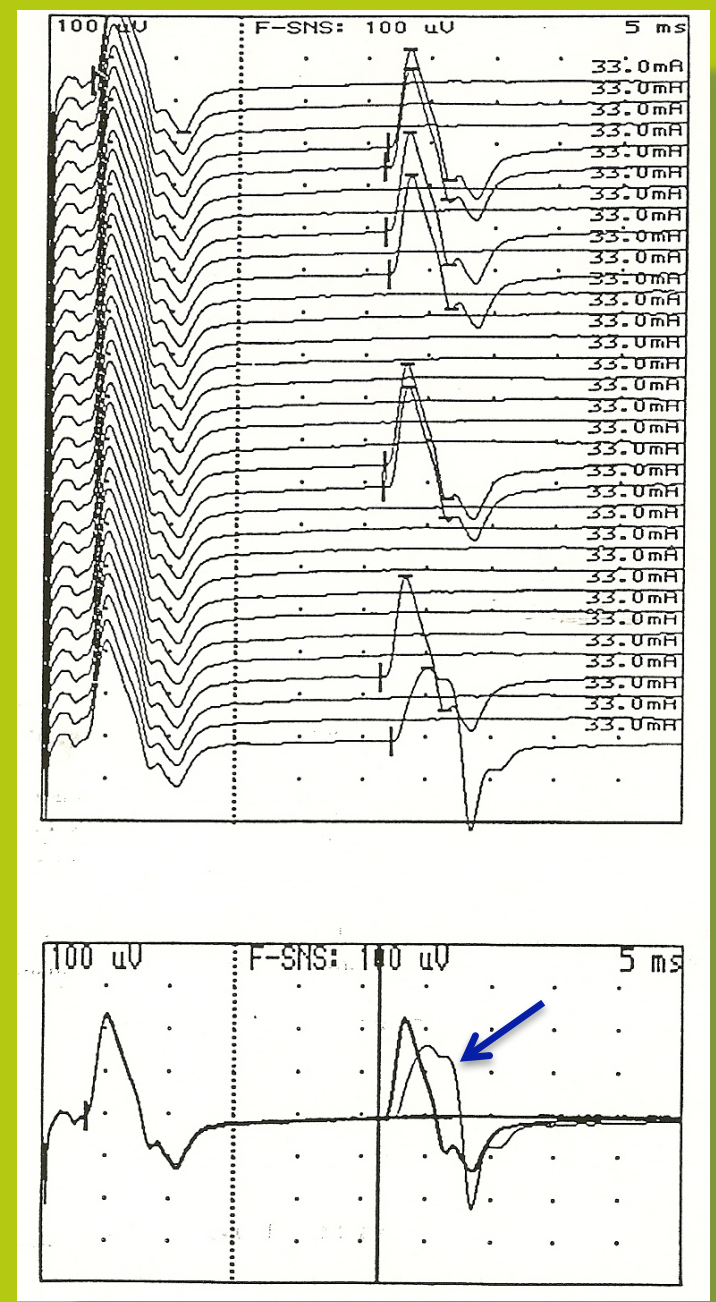

\section{Enregistrement de surface}

(1) réponse F unitaire

(2) réponses précoces et tardives de morphologie identique $=>$ un seul axone moteur alpha impliqué

(3) une réponse tardive de morphologie distincte de la réponse précoce $\Rightarrow$ activation d'un autre motoneurone par voie réflexe $=$ réflexe $\mathrm{H}$ 


\section{Doubles chocs serrés (ISI:10 ms) supramaximaux : abolition F1}
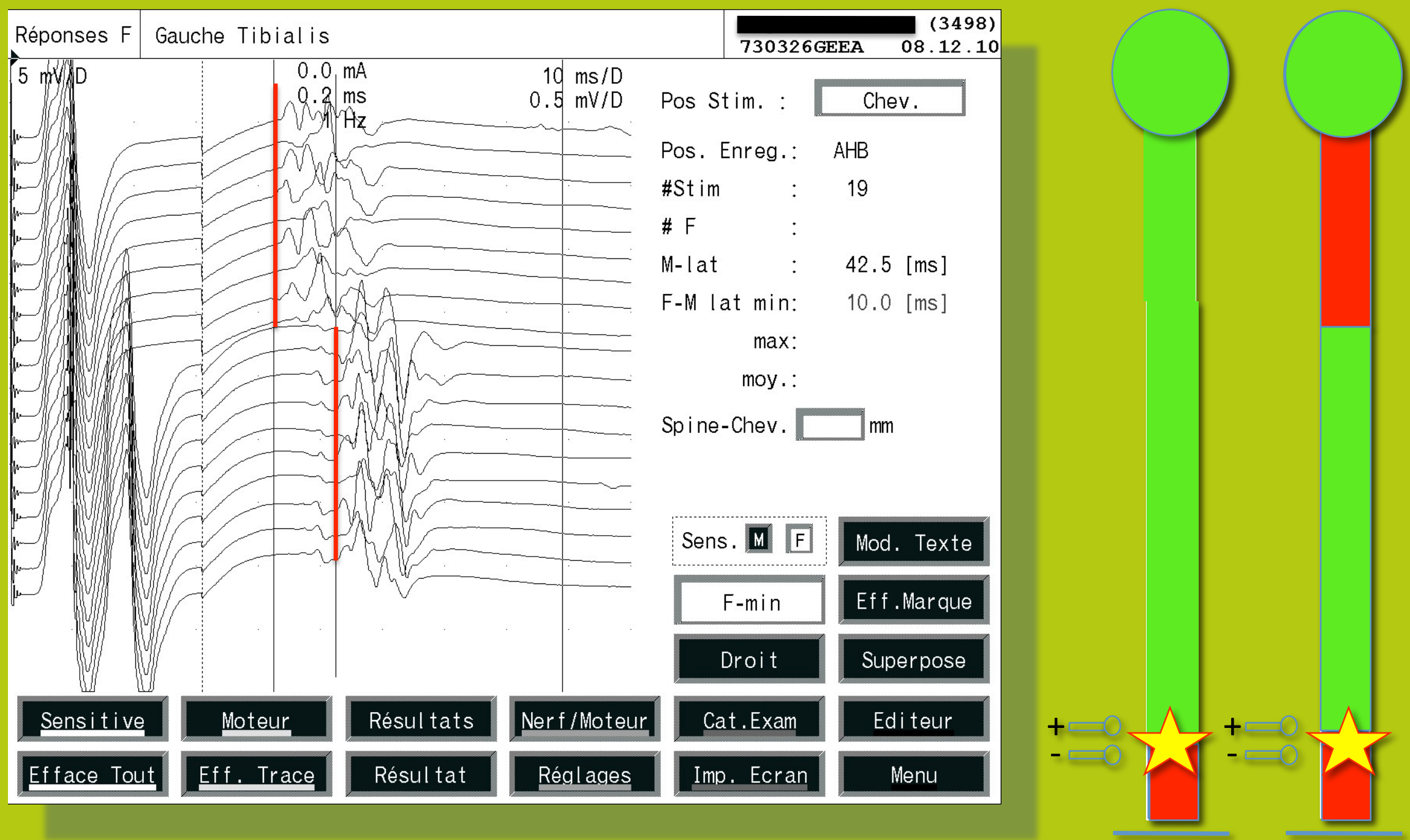


\section{doubles chocs serrés (ISI:10 ms) d'intensité croissante : H persiste}
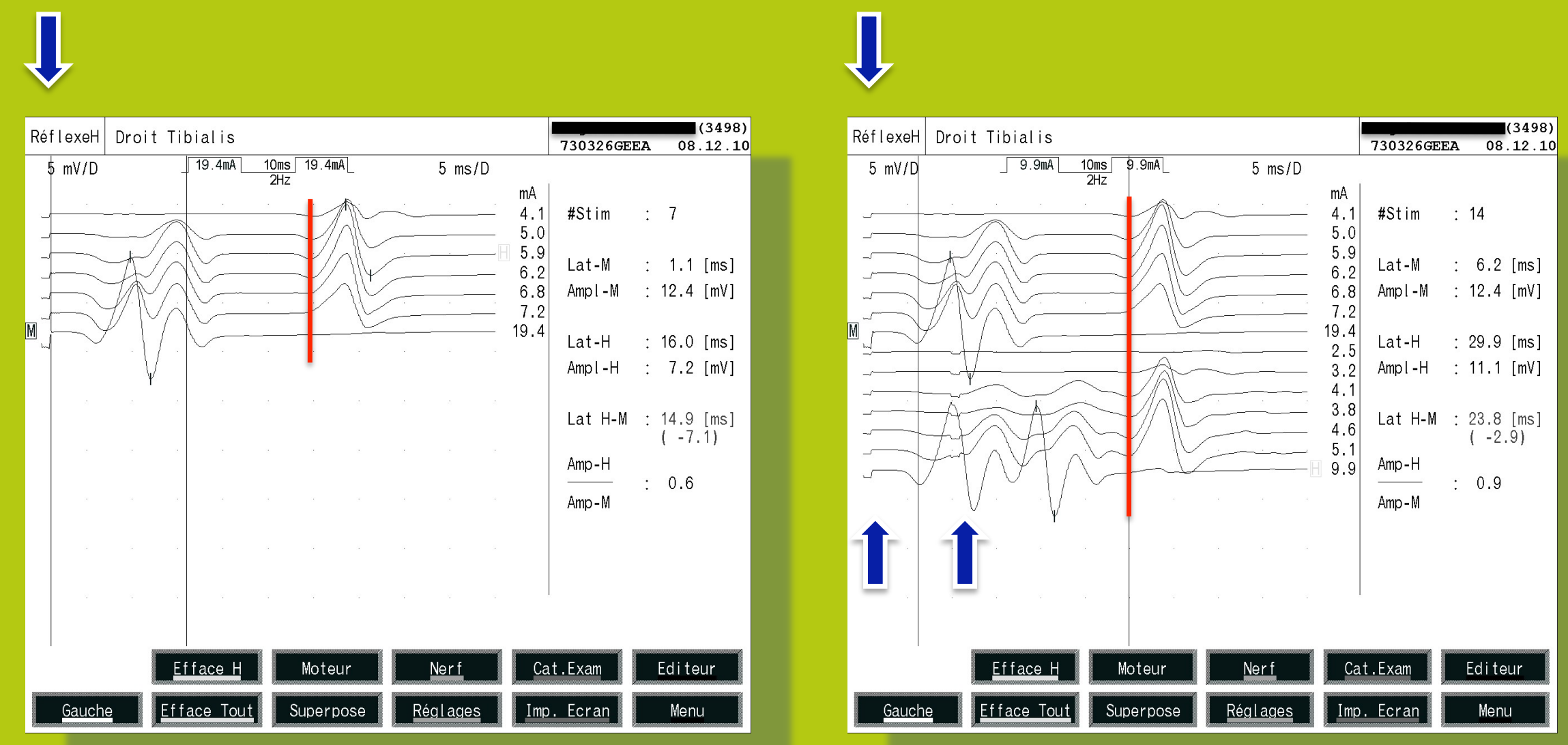


\section{Doubles chocs serrés (ISI:10 ms) supramaximaux : réflexe HH}
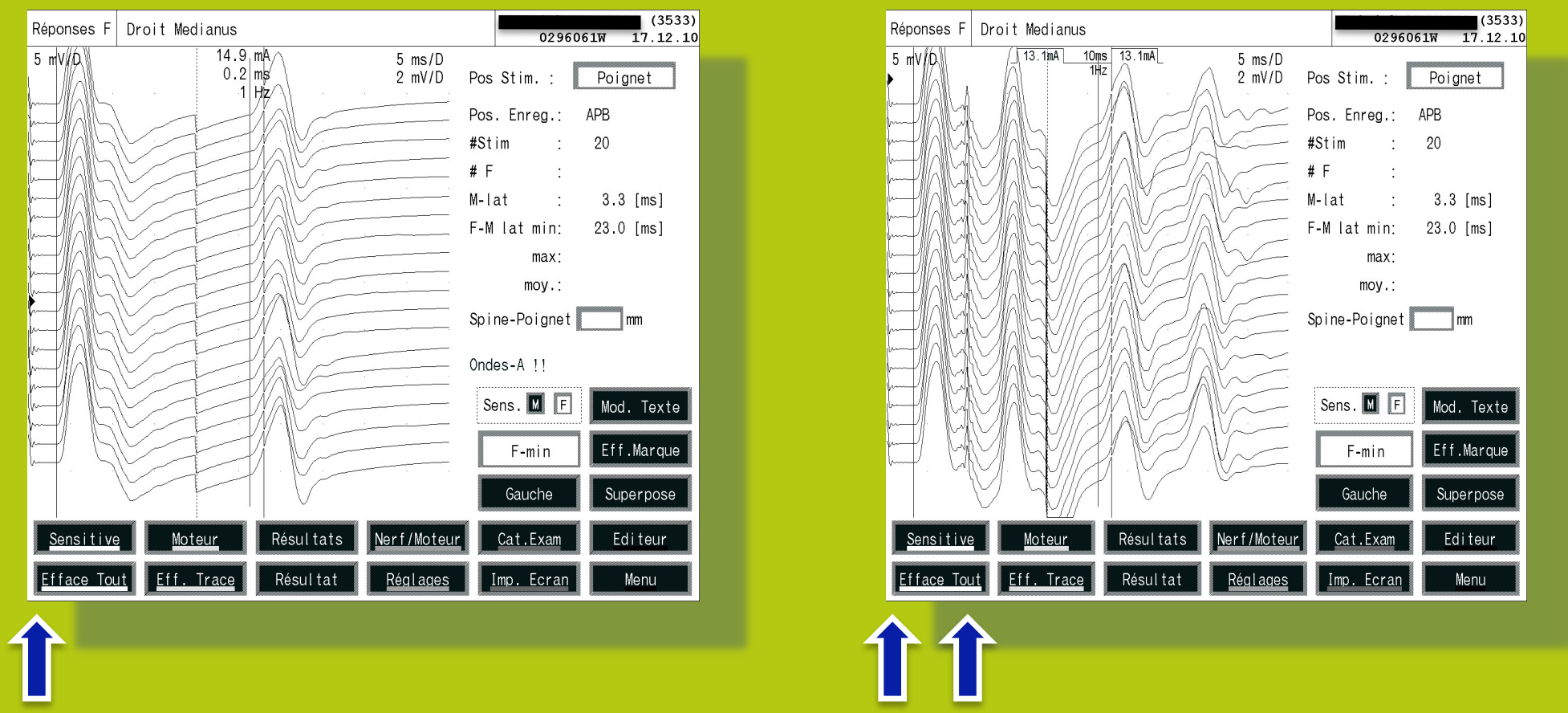

Réponses tardives d'amplitude variable

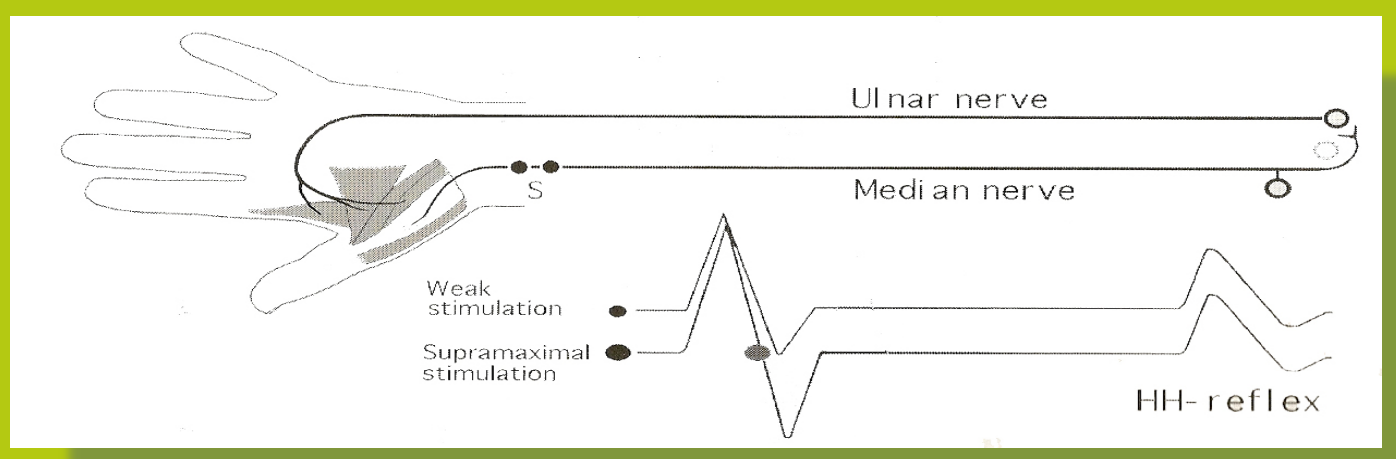




\section{Doubles chocs serrés (ISI:10 ms) : RAM persiste}
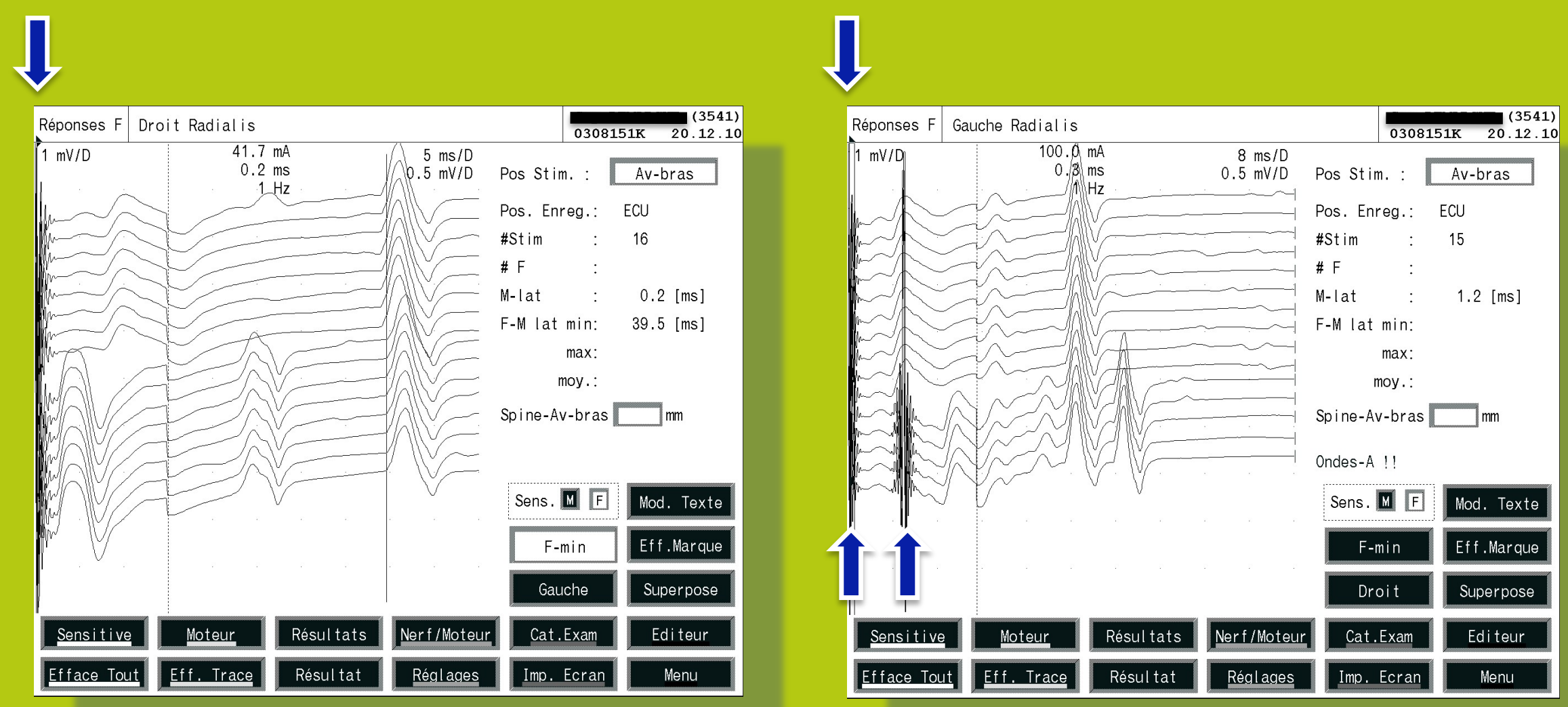

Réponses indirectes Morphologie constante Latence stable 


\section{Doubles chocs serrés (ISI:10 ms) : RAM persiste}
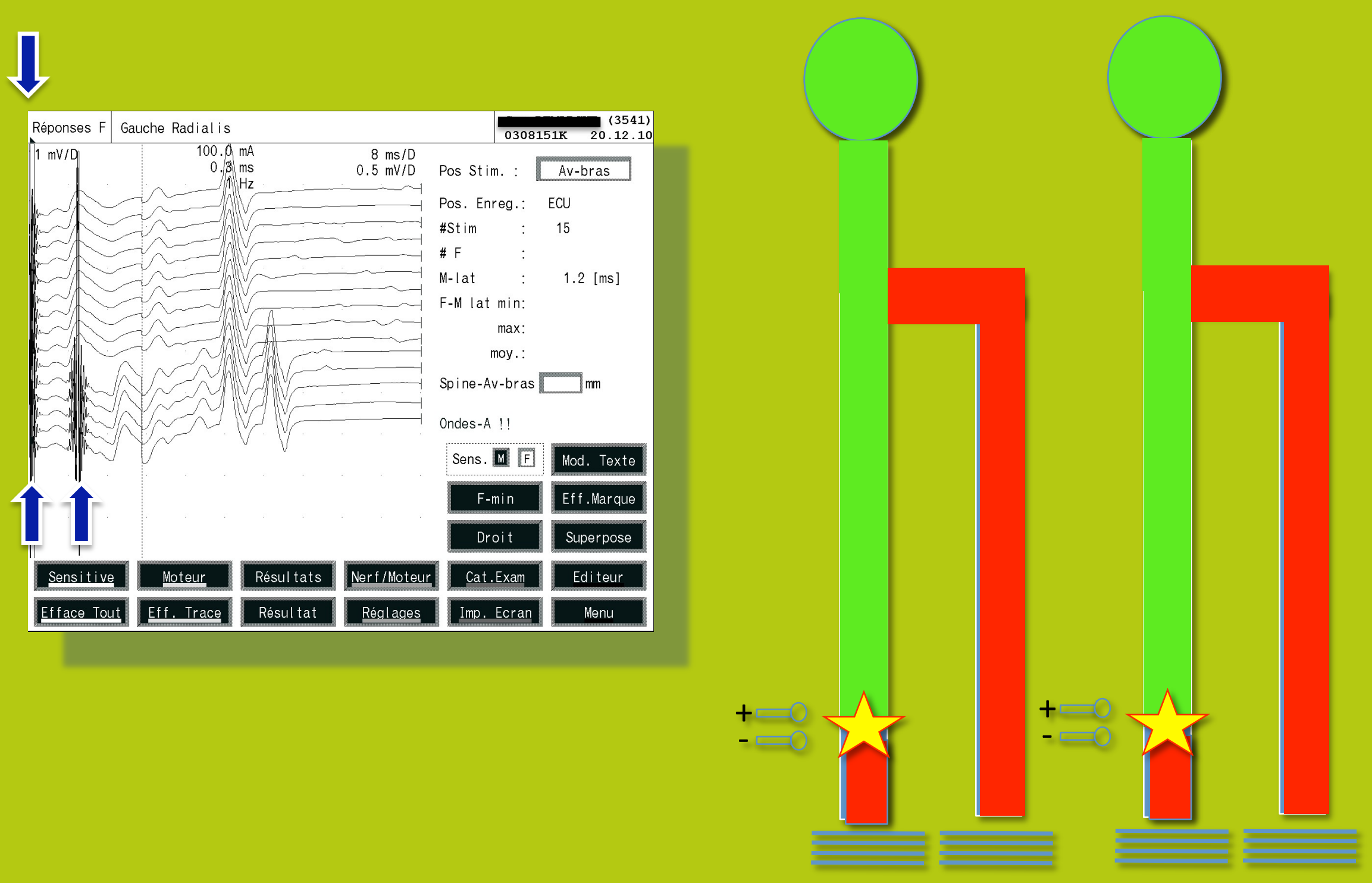


\section{Doubles chocs serrés (ISI:10 ms) : abolition de la DDI}

(1) comme pour la réponse $F$, l'afférence $=$ efférence

(2) $\Rightarrow$ réponse intermédiaire indirecte disparaissant lors de la double stimulation serrée

(3) $><$ réponse F

svt latence + courte pfs constante pfs instable morphologie identique ce n'est pas une onde spinale

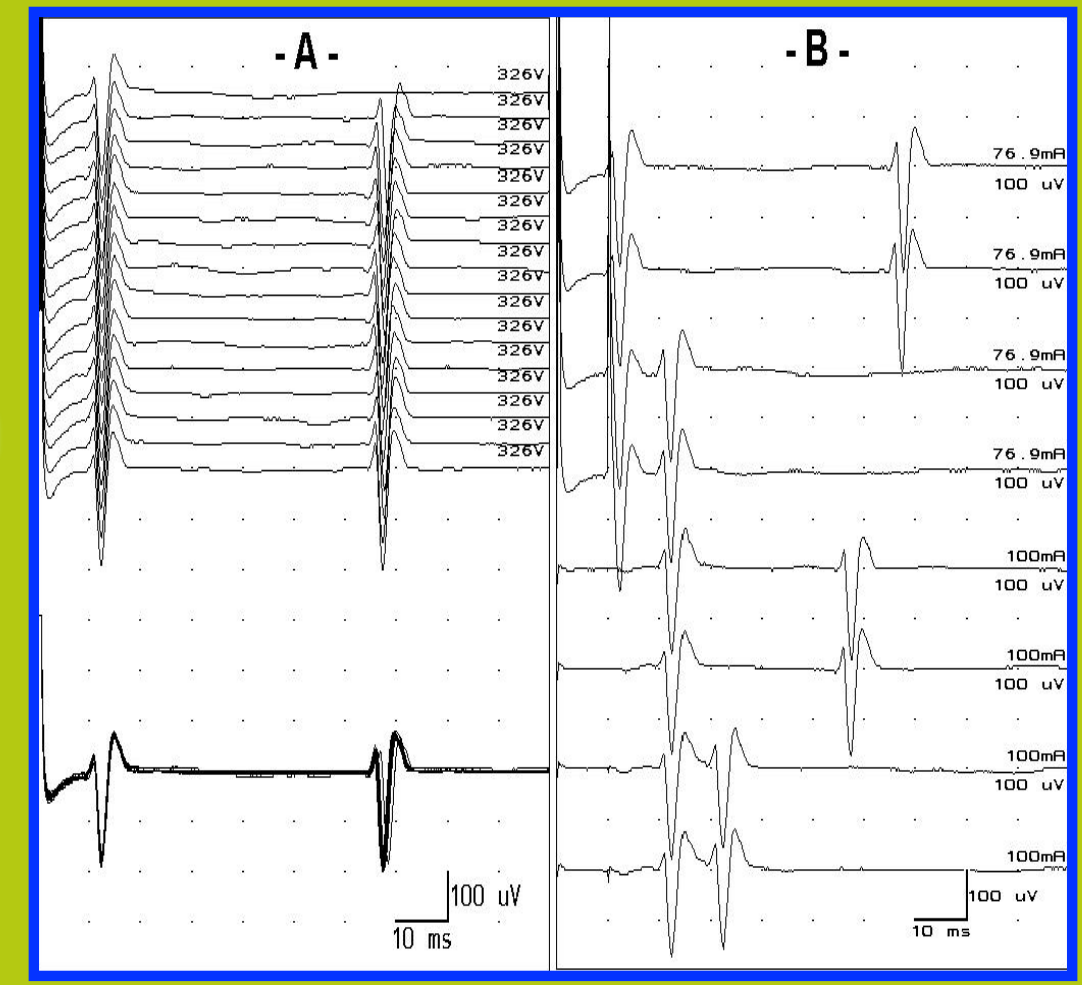

Soichot et Magistris, Liège 2004 


\section{Réflexe tardif}

(1) Stimulation nerf médian/détection thénarienne

(2) Latences (42,4 et $66,3 \mathrm{~ms}$ ) incompatibles avec une réponse $F$ normale : démyélinisation?

(3) L'absence de réponse $M$ suggère l'origine réflexe

(4) Circuit poly-synaptique complexe médullaire, bulbaire, voire transcortical

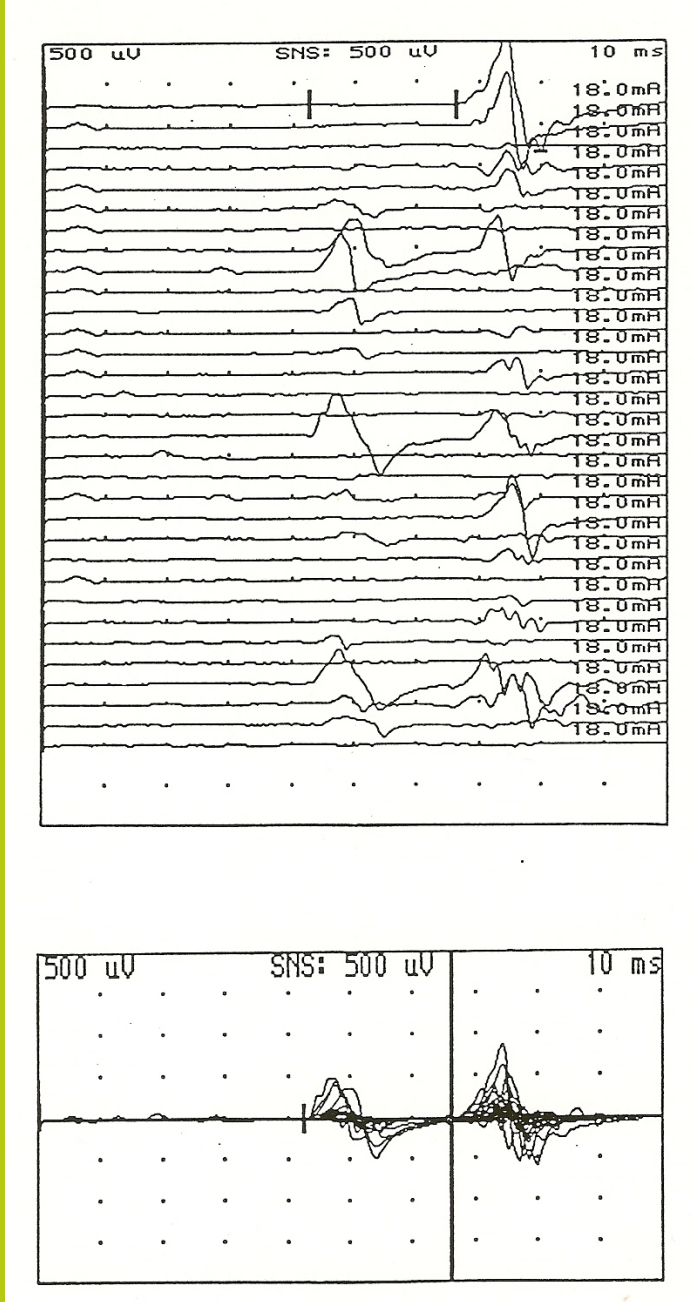




\section{États d'excitation}

Persistance des réponses $F$ États d'hypoexcitabilité : USI, sédatifs

Manceuvres de facilitation 


\section{Persistance des réponses F}

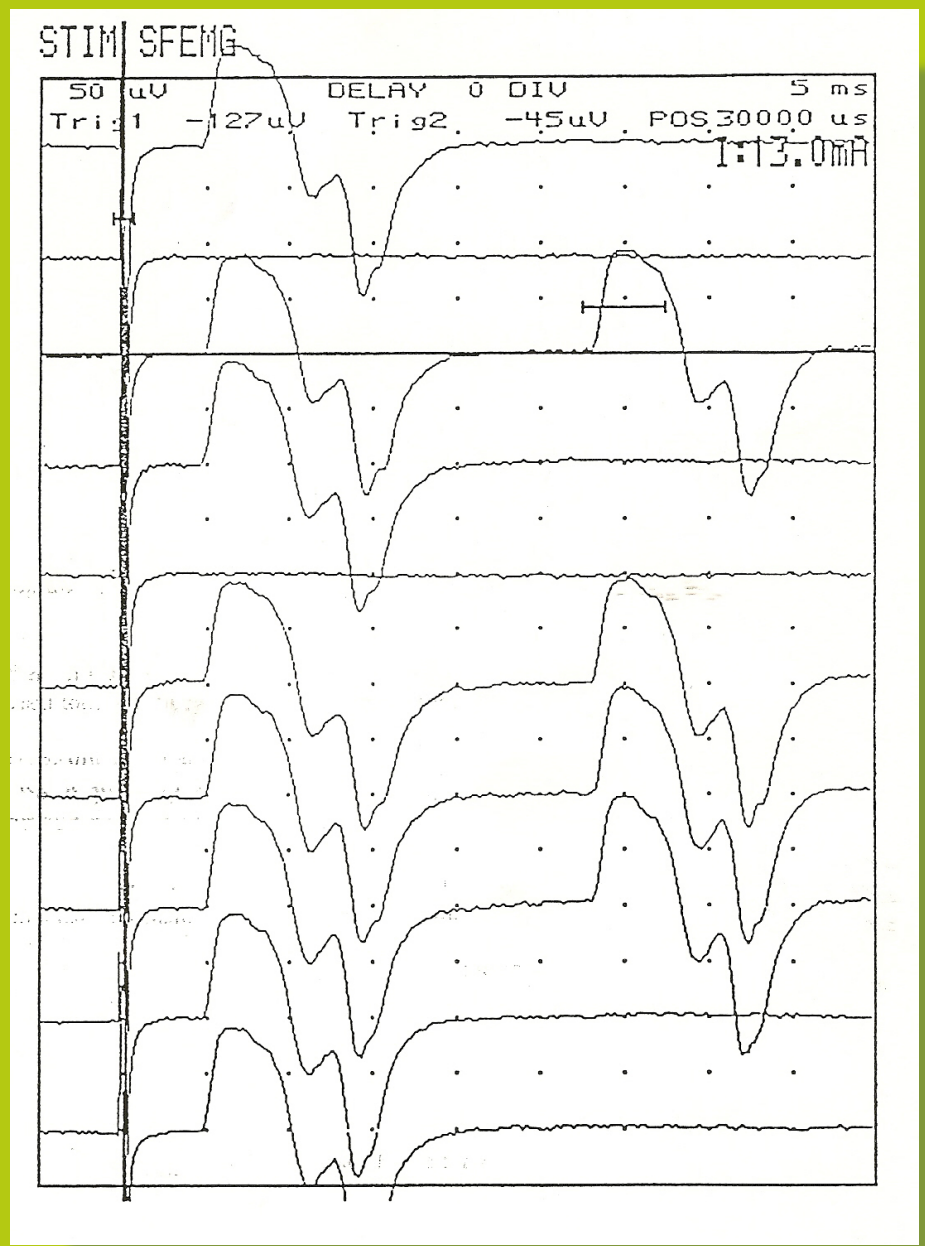

(1) Intensité du stimulus?

NON

(2) Fréquence du stimulus ?

NON (0,5-5 Hz, Schiller et Stålberg 1978)

(3) UM à conduction rapide?

NON (Kimura et al 1984)

OUI (Fischer 1982, 1985, 1988, 1992)

(4) UM à twitch élevé ?

NON sauf...(Dengler et al 1992) 


\section{Persistance des réponses $F$}

Excitabilité des motoneurones spinaux?

OUI : niveau d'excitabilité des MN, ajusté en permanence par les afférences inhilbitrices et activatrices, conditionne :

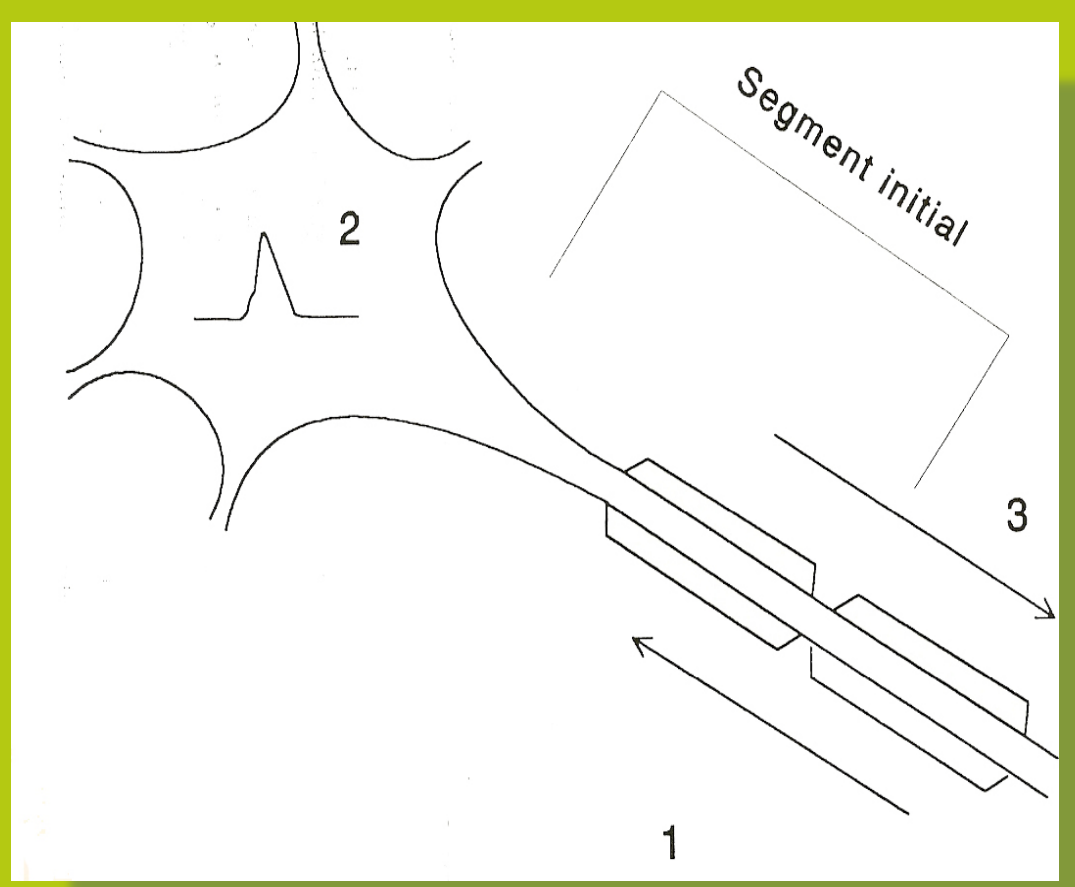

(1) passage des courants antidromiques à travers le SI (augmentation de surface membranaire non myélinisée) (Eccles 1955)

(2) délai pour que PA somatique dépolarise de nouveau le $\mathbf{S I}$ (Schiller et Stålberg 1978, Brown 1984)

(3) durée de la période réfractaire absolue du SI $(0,9-1,2 \mathrm{~ms}$, Eccles 1955) 


\section{États de manque (hypoexcitabilité)}

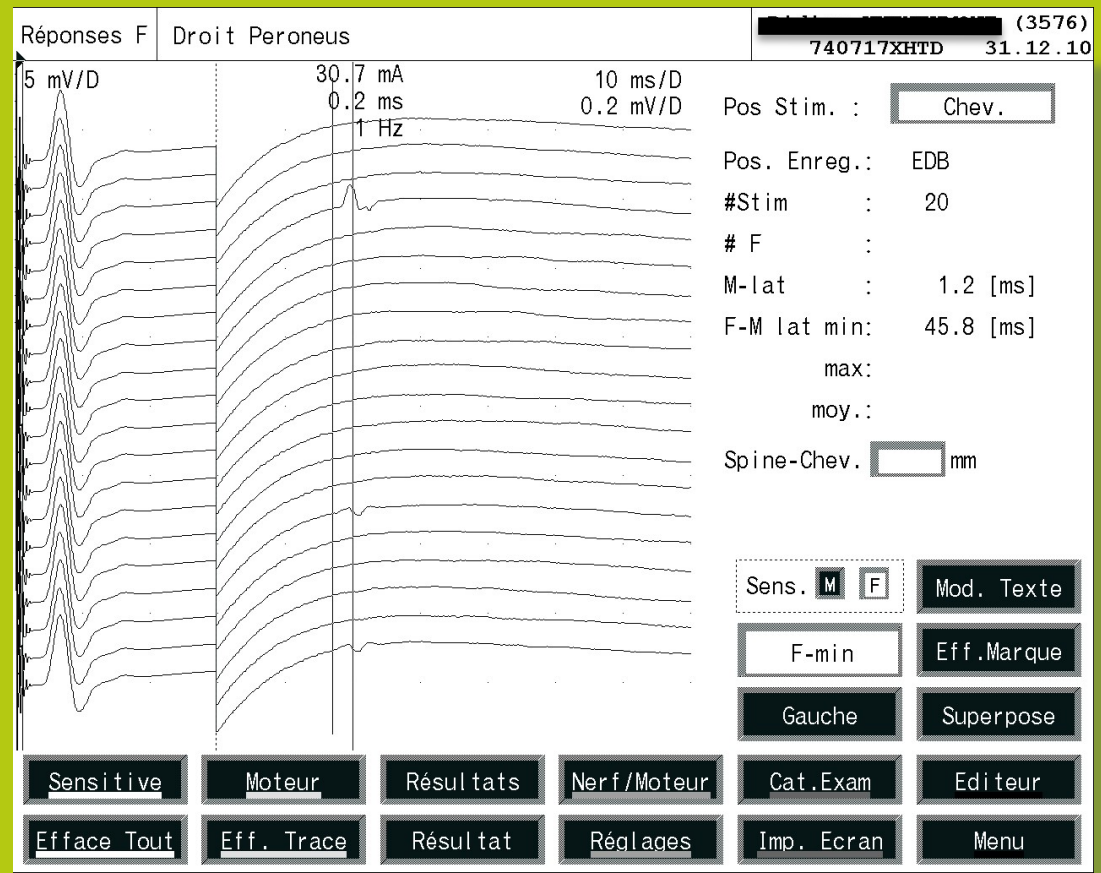

(1) tranquillisants

(2) sédation

(3) patient dans USI

(4) en dehors de toute atteinte neurologique

(5) $><$ atteinte proximale du SNP 


\section{Jendrassik ou équivalent}

(1) Influence les réponses spinales $\mathrm{H}$ et $\mathrm{F}$

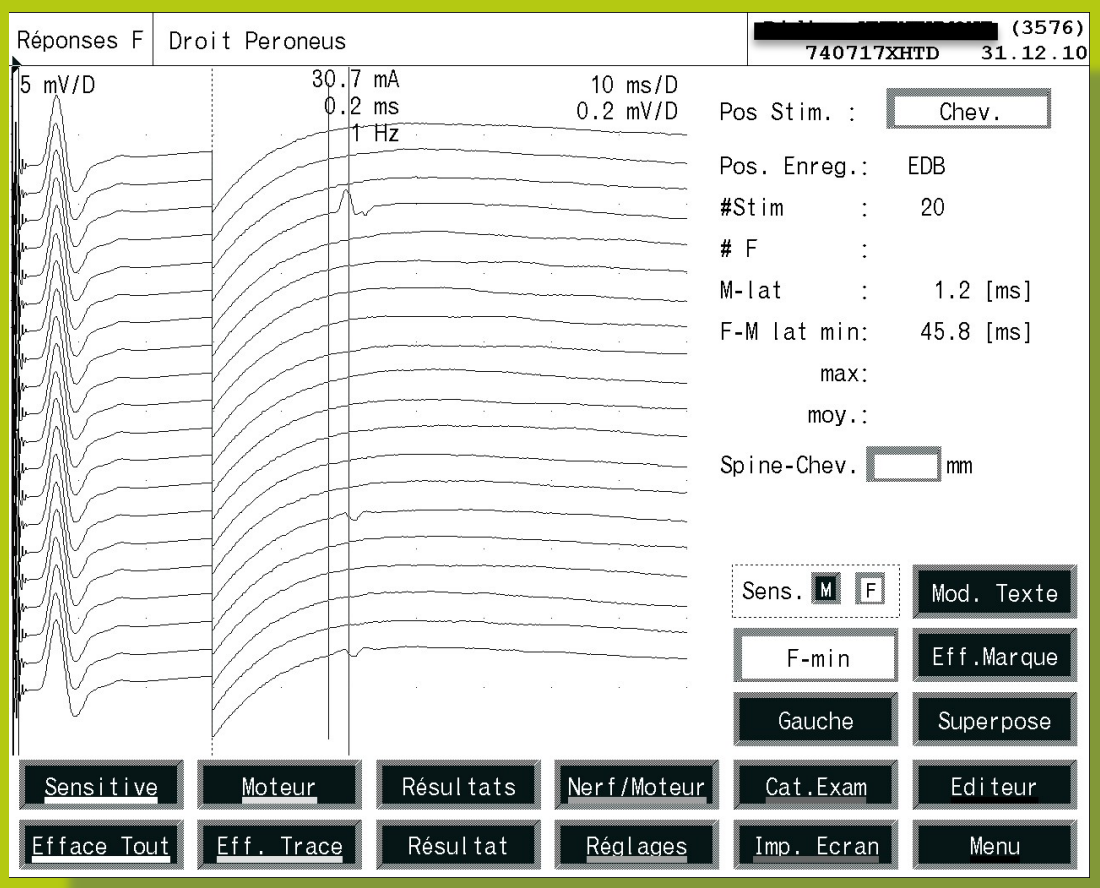

sans facilitation

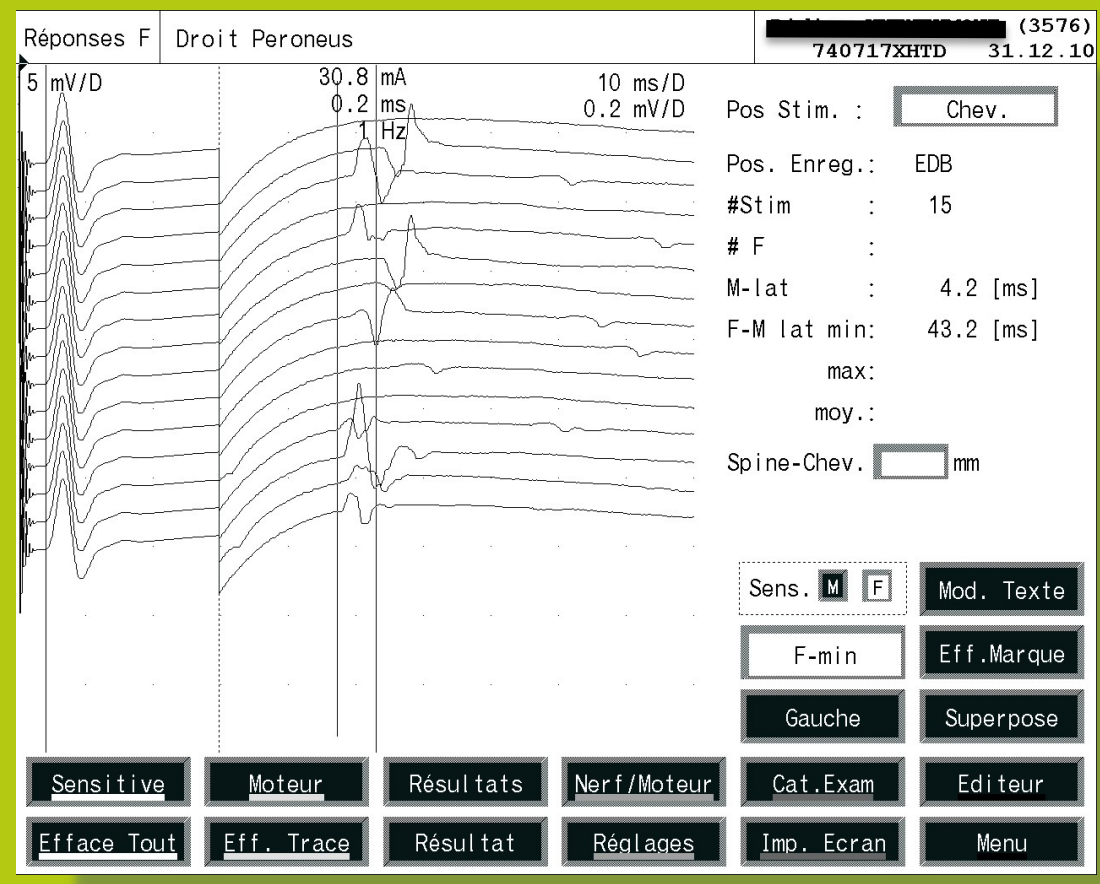

avec facilitation 


\section{Jendrassik ou équivalent}

(1) Influence les réponses spinales $\mathrm{H}$ et $\mathrm{F}$

(2) Sans effet sur les réponses intermédiaires RAM et DDI

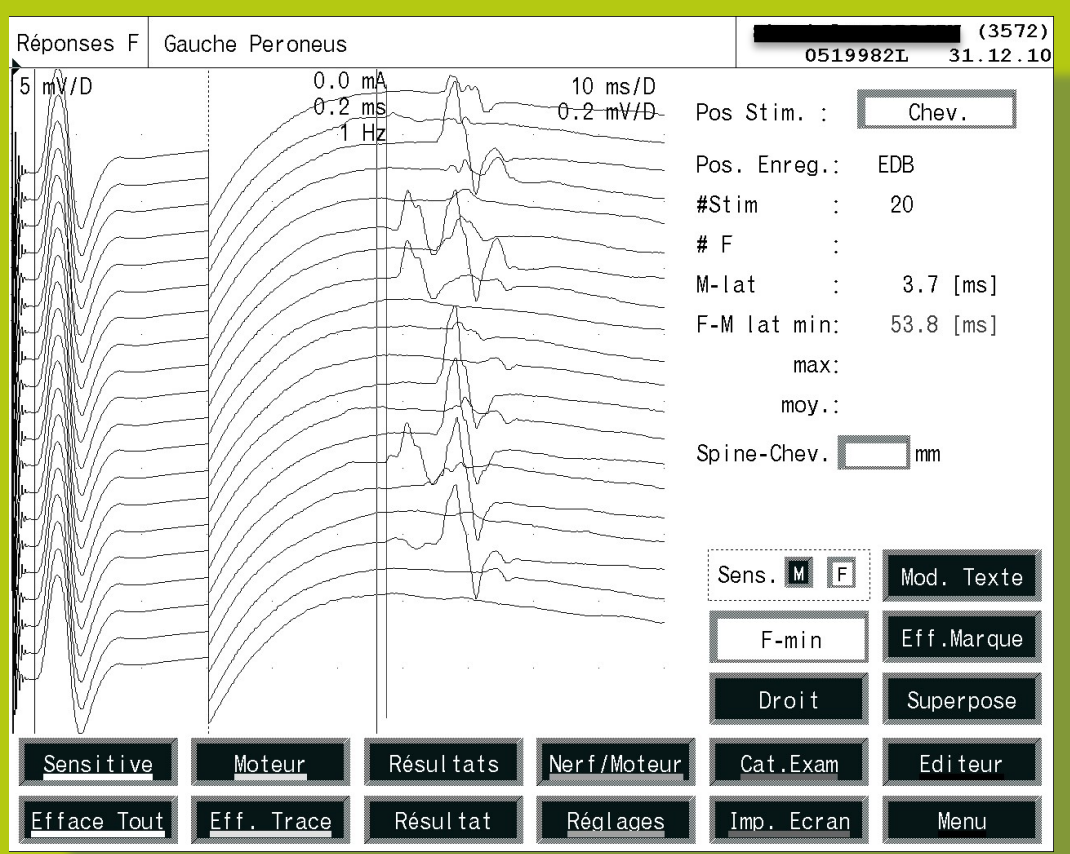

sans facilitation

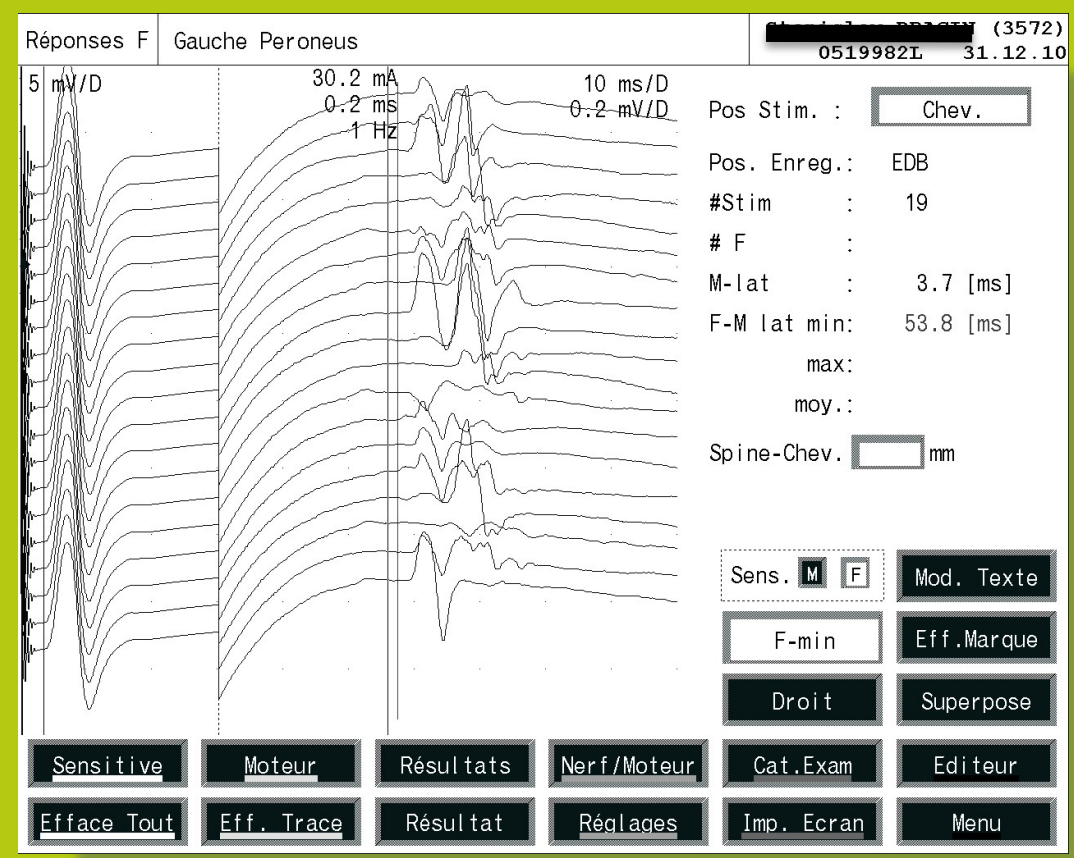

avec facilitation 


\section{États normaux}

Paramètres d'analyse Valeurs normatives 


\section{Méthodologie}

(1) Cathode à $8 \mathrm{~cm}$ de $\mathbf{G 1}$ (électrode de détection " active ")

- en ligne droite pour les nerfs ulnaires, fibulaire et tibial

- en respectant le trajet nerveux pour le nerf médian

(2) G2 (référence)

- pouce (n. médian), auriculaire (n. ulnaire), gros orteil (n. tibial)

- en arrière de la malléole externe (n. fibulaire)

(3) Réglages identiques à ceux d'une conduction motrice distale

- intensité (supramaximale), durée de stimulation $(0,2 \mathrm{~ms})$, fréquence de stimulation (1 Hz max.), filtres ( $2 \mathrm{~Hz}-10 \mathrm{KHz}$ )

(4) Latence minimale mesurée si 7 réponses $F$ au minimum 


\section{Paramètres d'analyse}

(1) Latence : minimale, moyenne, maximale, maximale-minimale, F-ratio, F-ratio modifié

(2) Vitesse de conduction : minimale, moyenne, maximale

(3) Amplitude : moyenne, maximale, absolue ou relative à la réponse $M$ maximale

(4) Durée : moyenne

(5) Persistance : globale ou des ondes F monomorphes 
Article original

Symétrie et reproductibilité temporelle des données neurographiques

Symmetry and temporal variability of neurography

O. Bouquiaux, A. Horward, F.C. Wang *

Département d'electroneuromyographie, service de médecine de l'appareil locomoteur, CHU Sart-Tilman, batiment B35, 4000 Liège, Belgique

\begin{tabular}{|l|c|c|c|c|c|c|c|c|}
\hline & & $\begin{array}{l}\text { Amplitude } \\
\text { PGAM }\end{array}$ & $\begin{array}{l}\text { Surface } \\
\text { PGAM }\end{array}$ & VCM & LDM & $\begin{array}{l}\text { F-Lat } \\
\text { min }\end{array}$ & $\begin{array}{l}\text { F-Lat } \\
\text { moy }\end{array}$ & F-Amp \\
\hline Médian: & G/Dr & 11 & 13 & & 6 & $\mathbf{3}$ & $\mathbf{3}$ & 44 \\
& T1/T2 & 9 & 13 & & 6 & $\mathbf{2}$ & $\mathbf{2}$ & 40 \\
\hline Ulnaire: & G/Dr & 10 & 9 & & 7 & $\mathbf{3}$ & $\mathbf{2}$ & 38 \\
& T1/T2 & 6 & 9 & & 5 & $\mathbf{2}$ & $\mathbf{2}$ & 38 \\
\hline Fibulaire: & G/Dr & 14 & 17 & 4 & 6 & $\mathbf{3}$ & 3 & 66 \\
& T1/T2 & 10 & 16 & 4 & 5 & $\mathbf{3}$ & $\mathbf{3}$ & 46 \\
\hline Tibial: & G/Dr & 14 & 13 & & 7 & $\mathbf{2}$ & & 26 \\
& T1/T2 & 8 & 13 & & 8 & $\mathbf{2}$ & & 34 \\
\hline
\end{tabular}


Reproducibility in nerve conduction studies and F-wave analysis

Denise Spinola Pinheiro, Gilberto Mastrocola Manzano, João Antonio Maciel Nóbrega* Federal University of Sāo Paulo, Neurology and Neurosurgery Department, Setor de Pesquisa e Pós-graduaçāo em Neurofisiologia Clínica (SEPENC). Accepted 16 May 2008

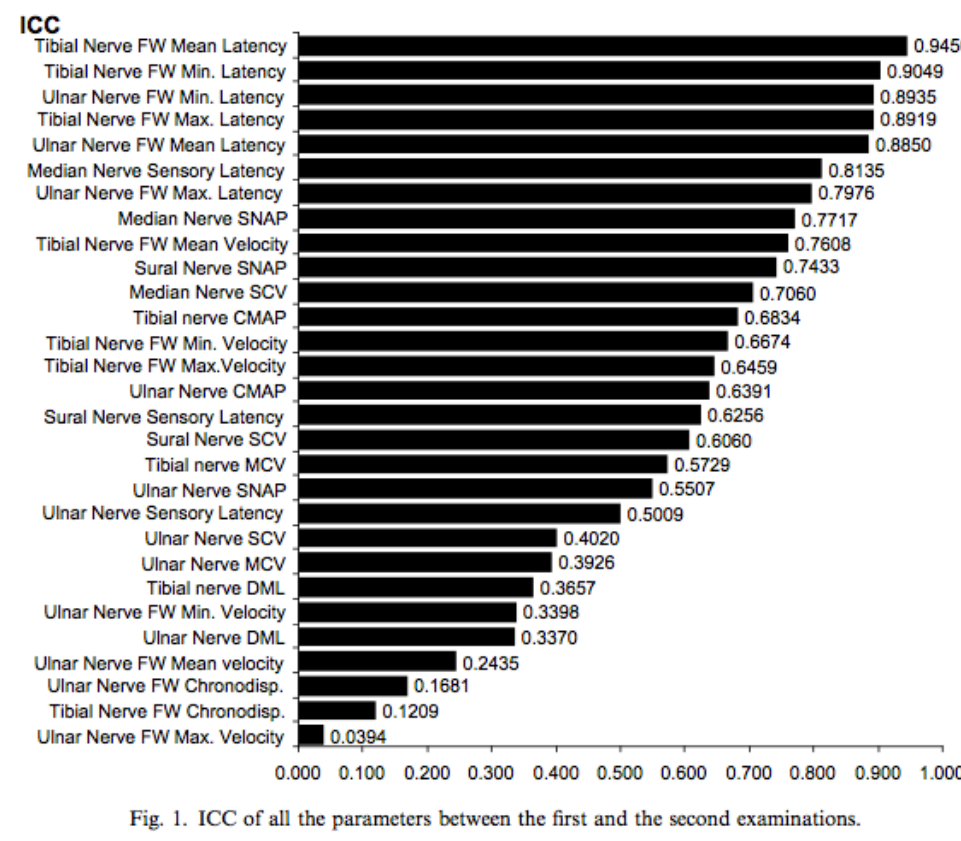

]$_{0.1681}^{0.2435}$

Fig. 1. ICC of all the parameters between the first and the second examinations.

0.7060

0.5507

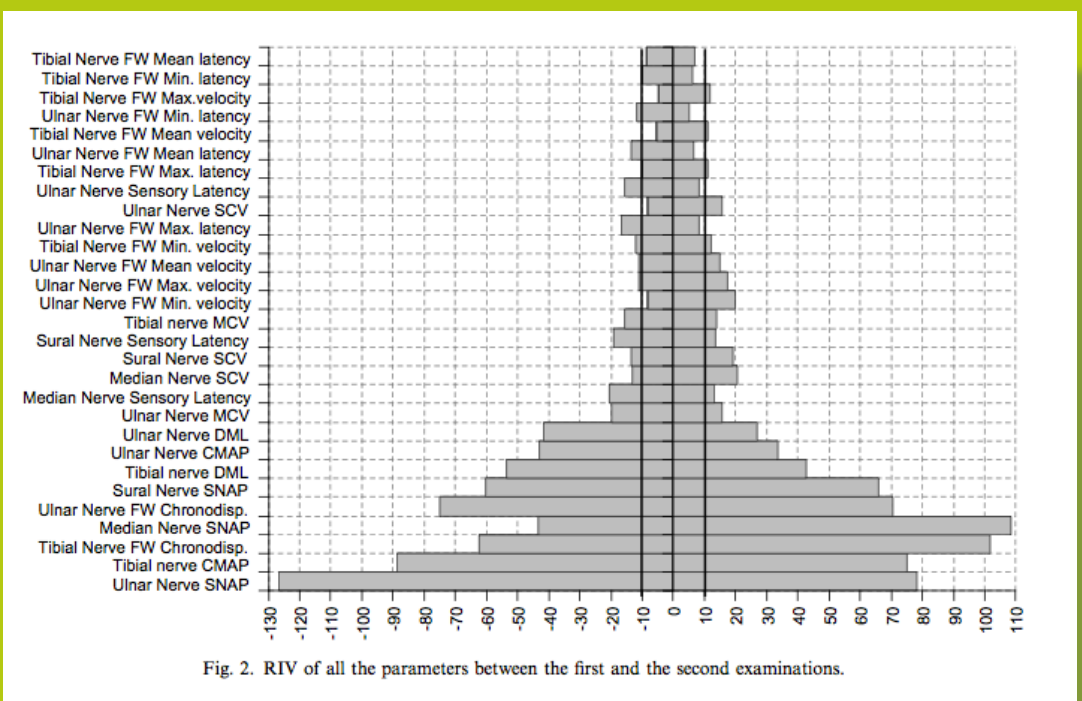

RIV of all the Pats 


\section{Valeurs normatives absolues}

analyse multivariée $=>$ latence des ondes $\mathrm{F}$ corrélée:

- chez l'adulte : taille, âge, genre (parfois) (Puksa et al, 2003)

- chez l'enfant (3-20 ans) : taille (Puksa et al, 2011)

Équations de régression linéaire multiple:

$$
\begin{aligned}
& \mathbf{Y}=\text { constante }+\mathbf{X}_{1}{ }^{*} \text { age }+\mathbf{x}_{2}{ }^{*} \mathrm{age}^{2}+\mathbf{X}_{3}{ }^{*} \text { taille } \\
& \mathbf{Y}=\text { valeur de référence moyenne atiendue }
\end{aligned}
$$

Z-score (l'écart de la valeur mesurée par rapport Y) :

$$
\mathbf{Z}=(\mathbf{Y} \text {-valeur mesurée }) / \mathrm{DS}
$$

Z-score considéré normal : entre -2 et 


\section{Valeurs normatives absolues}

ABSTRACT: In this study we examined the diagnostic sensitivity of minimal F-wave latency, F-wave persistence, motor nerve conduction velocity (MCV), and amplitude of the compound motor action potential (CMAP) of the median, ulnar, tibial, and peroneal nerves, and of sensory conduction velocity (SCV) and sensory nerve action potential (SNAP) amplitude of the sural nerve in 82 diabetic patients. For the median, ulnar, and tibial nerves the $Z$ scores of the minimal $F$-wave latency were significantly larger than those of the MCV, and for all four motor nerves the $Z$ scores of the minimal $F$-wave latency were significantly larger than those of the amplitude of the CMAP. The $Z$ scores of the peroneal minimal F-wave latency exceeded those of peroneal MCV, sural SCV, and sural SNAP. F-wave persistence did not differ significantly from the reference values. In conclusion, minimal $\mathrm{F}$ wave latency is the most sensitive measure for detection of nerve pathology and should be considered in electrophysiological studies of diabetic patients. @ 1997 John Wiley \& Sons, Inc. Muscle Nerve 20: 1296-1302, 1997

Key words: minimal F-wave latency; nerve conduction; diabetic polyneuropathy; diagnostic sensitivity; nerve pathology

\section{F-WAVE LATENCY, THE MOST SENSITIVE NERVE CONDUCTION PARAMETER IN PATIENTS WITH DIABETES MELLITUS}

HENNING ANDERSEN, MD, ${ }^{1,2 \star}$ ERIK STÅLBERG, MD, PhD, ${ }^{1}$ and BJÖRN FALCK, MD, PhD'

${ }^{1}$ Department of Clinical Neurophysiology, Un iversity Hospital, Uppsala, Sweden

${ }^{2}$ Department of Neurology, Aarhus University Hospital, 8000 Aarhus, Denmark

Received 27 March 1996; accepted 20 April 1997 


\section{Valeurs normatives relatives Limites de symétrie et de variabilité temporelle}

\begin{tabular}{|l|l|l|}
\hline Nerfs & Variabilité G/Dr & Variabilité temporelle \\
\hline Médian & $1,5 \mathrm{~ms} \mathrm{(1)}$ & $1,0 \mathrm{~ms}(1)$ \\
& $1,3 \mathrm{~ms}(2)$ & $1,0 \mathrm{~ms}(2)$ \\
\hline Ulnaire & $1,5 \mathrm{~ms}(1)$ & $1,2 \mathrm{~ms}(1)$ \\
& $1,4 \mathrm{~ms}(2)$ & $1,1 \mathrm{~ms}(2)$ \\
\hline Fibulaire & $3,0 \mathrm{~ms}(1)$ & $2,6 \mathrm{~ms}(1)$ \\
& $3,5 \mathrm{~ms}(2)$ & $3,7 \mathrm{~ms}(2)$ \\
\hline Tibial & $3,0 \mathrm{~ms}(1)$ & $2,0 \mathrm{~ms}(1)$ \\
& $3,0 \mathrm{~ms}(2)$ & $3,5 \mathrm{~ms} \mathrm{(2)}$
\end{tabular}

(1): Puksa et al, 2003 ; (2) : Bouquiaux et al, 2003 


\section{F-ratio}

\section{FLAT-PLAT- 1}

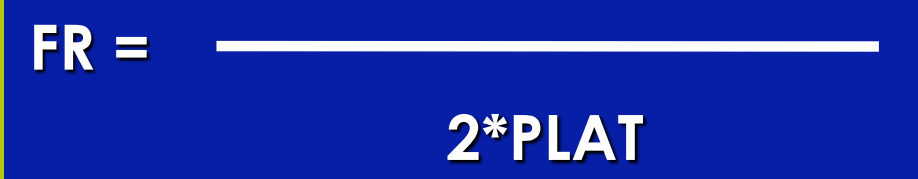

PLAT

\begin{tabular}{|l|l|}
\hline & Moyenne \pm DS \\
\hline Médian & $0,89 \pm 0,060$ \\
\hline Ulnaire & $0,91 \pm 0,059$ \\
\hline Fibulaire & $1,00 \pm 0,080$ \\
\hline Tibial & $1,17 \pm 0,063$ \\
\hline
\end{tabular}




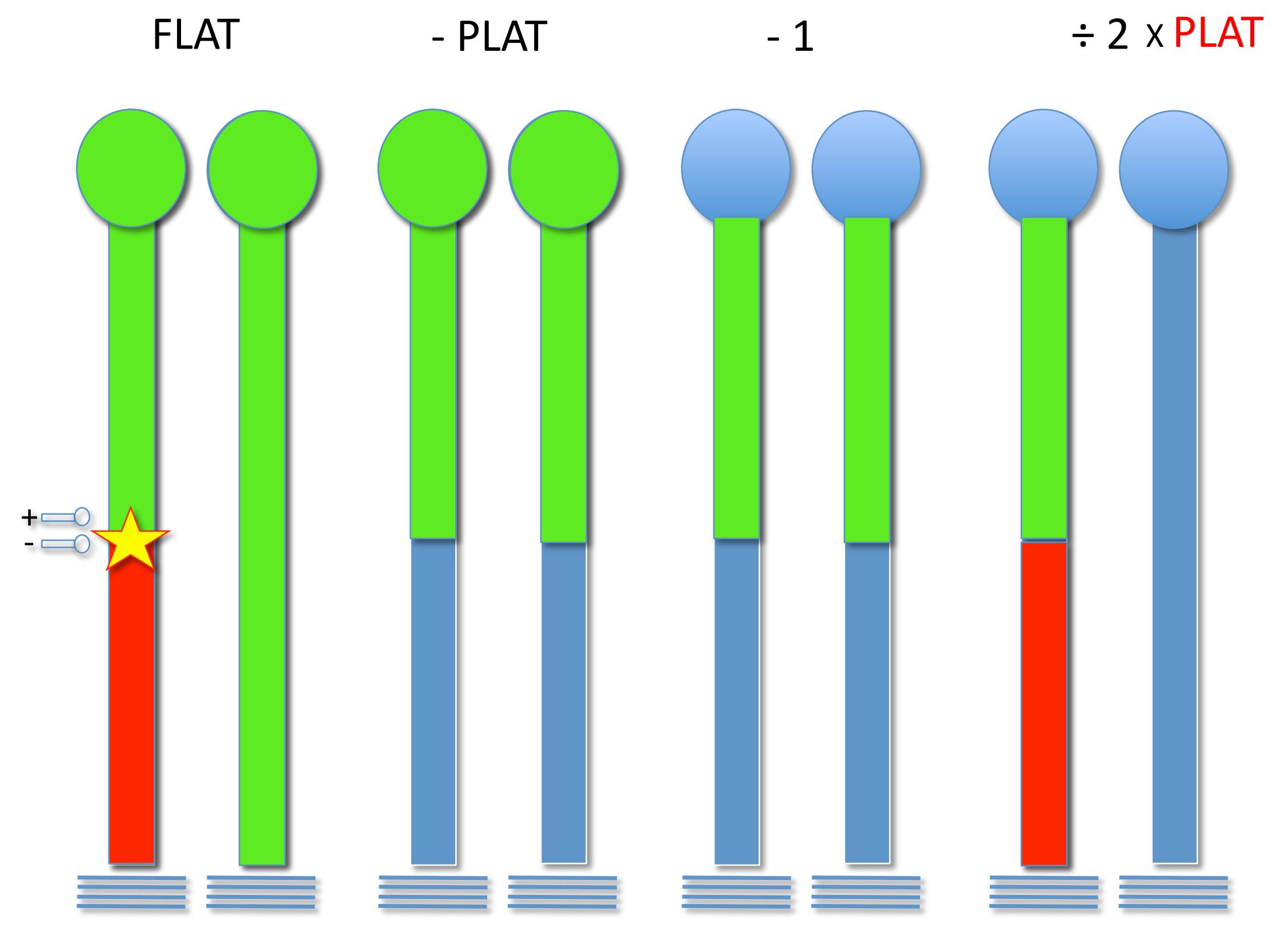




\title{
F-ratio modifié
}

\author{
(FLAT+DLAT -1)-2*PLAT
}

MFR =

2*DLAT

Moyenne \pm DS

Médian $1,92 \pm 0,20$

Ulnaire $\quad 2,86 \pm 0,27$

Fibulaire $3,50 \pm 0,40$

Tibial $\quad 3,30 \pm 0,37$ 


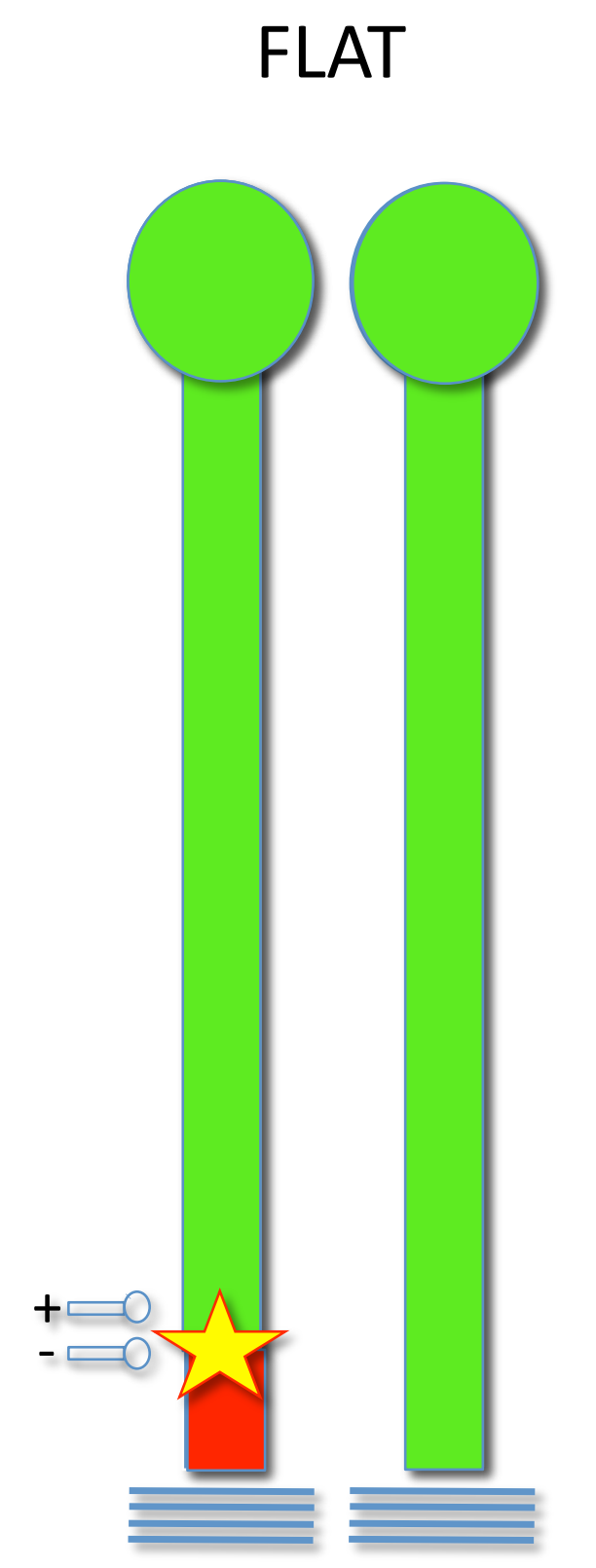

+ DLAT - 1

- 2 PLAT

$\div 2$ DLAT
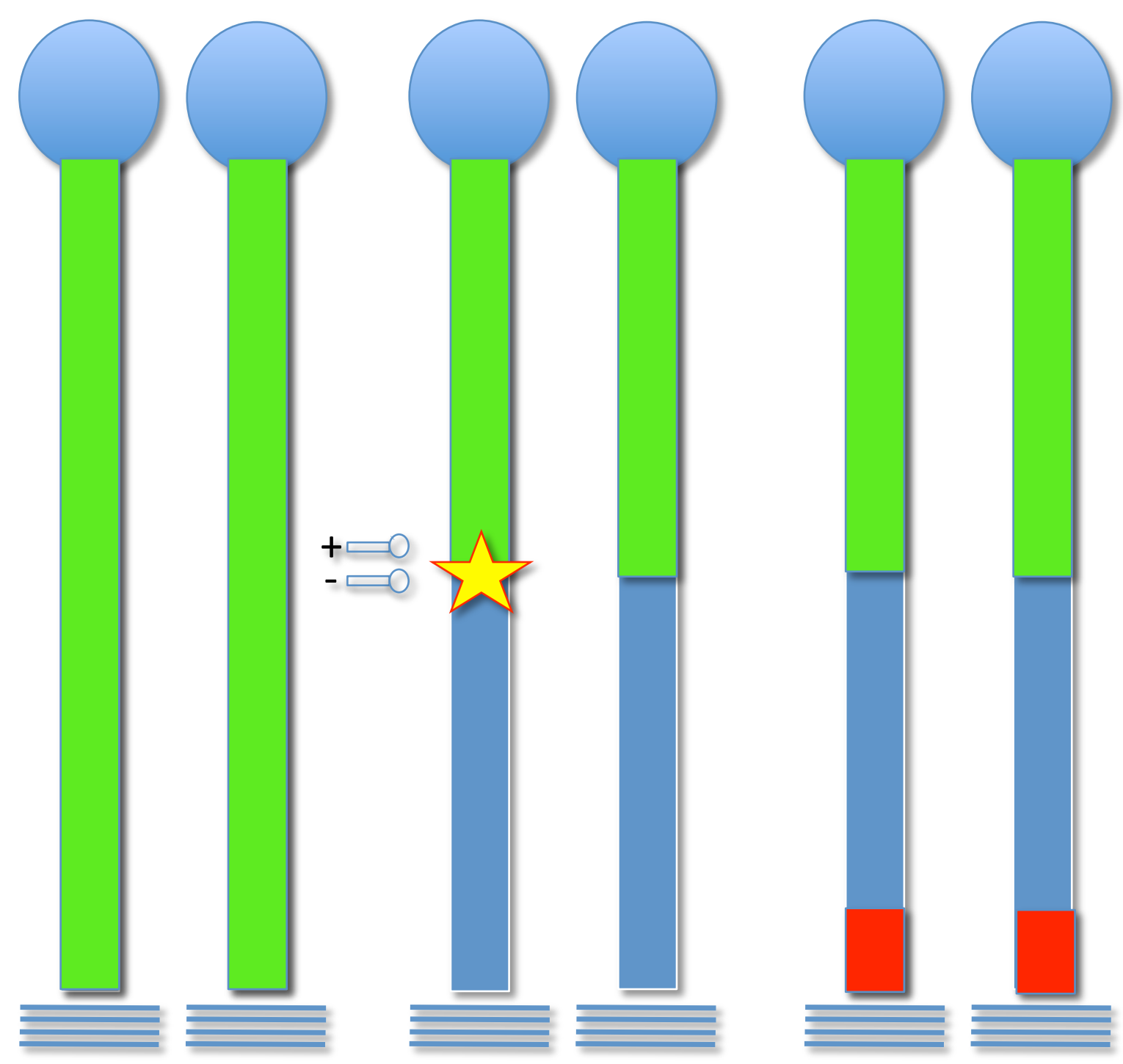


\section{États de mal}

Neuropathies démyélinisantes

Pathologies du motoneurone 


\section{Normal}
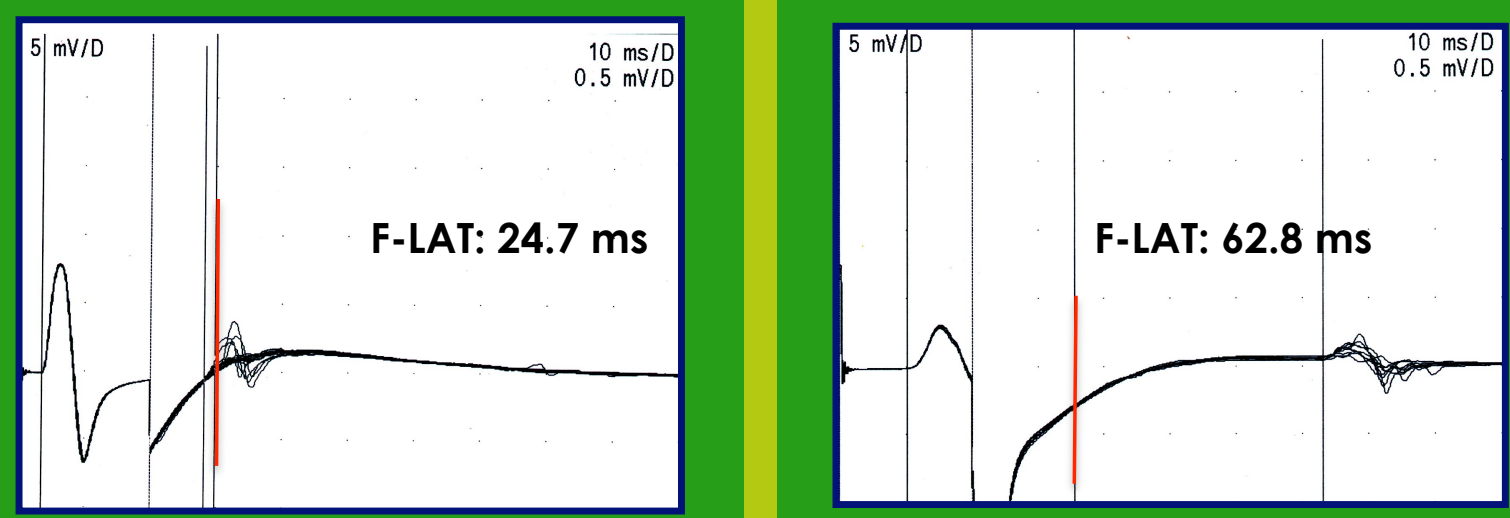

\section{CMTla}
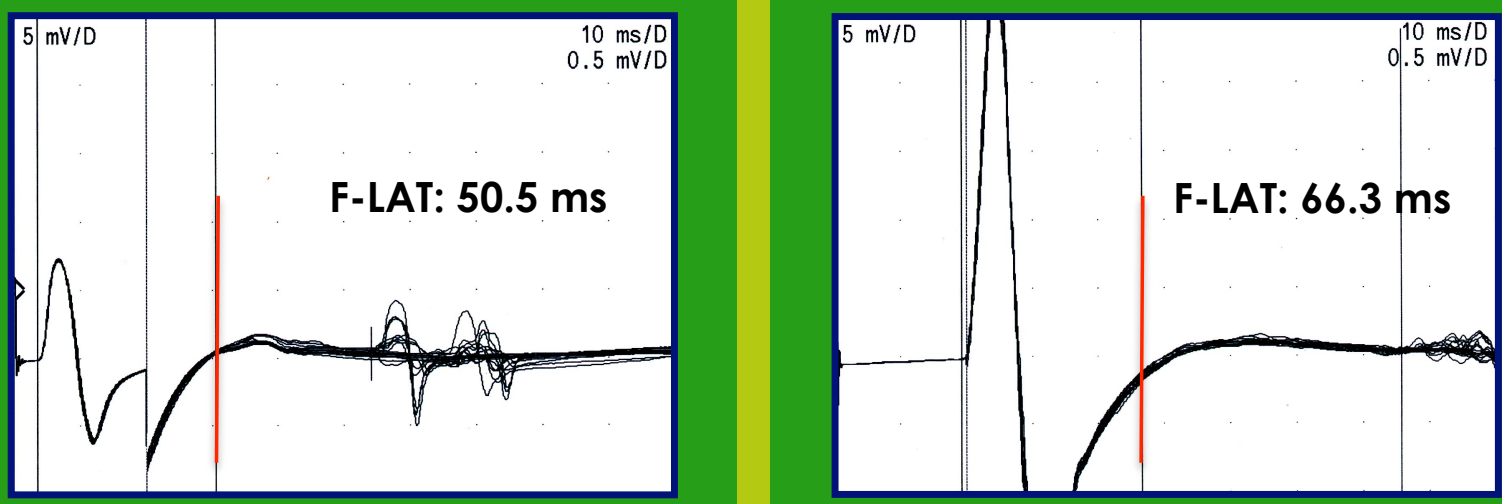

DADS 


\section{Neuropathies démyélinisantes}

\begin{tabular}{|l|l|l|l|}
\hline & CMT1a & PRNC & DADS \\
\hline $\begin{array}{l}\text { Index de } \\
\text { latence } \\
\text { terminale }\end{array}$ & $0.37(-1.39$ DS) & $\mathbf{0 . 5 1}(+2.5$ DS $)$ & $\mathbf{0 . 1 7}$ (-6.9 DS) \\
\hline F-ratio & $0.78(-1.80$ DS $)$ & & $\mathbf{0 . 5 7}$ (-5.3 DS) \\
\hline F-ratio modifié & $1.79(-0.65$ DS) & $\mathbf{4 . 7 5}(+14.2$ DS) & $\mathbf{0 . 8 8}$ (-5.2 DS) \\
\hline
\end{tabular}




\section{Latence F : 120\% LSN (n. tibial)}

\begin{tabular}{|l|l|l|l|l|l|l|}
\hline & $\mathbf{1 5 0}$ & $\mathbf{1 6 0}$ & $\mathbf{1 7 0}$ & $\mathbf{1 8 0}$ & $\mathbf{1 9 0}$ & $\mathbf{2 0 0}$ \\
\hline $\mathbf{2 0}$ & 58,4 & 64,1 & 69,7 & 75,4 & 80,9 & 86,5 \\
\hline $\mathbf{3 0}$ & 59,9 & 65,5 & 71,2 & 76,8 & 82,4 & 88,1 \\
\hline $\mathbf{4 0}$ & 61,4 & $\mathbf{6 7 , 1}$ & $\mathbf{7 2 , 7}$ & $\mathbf{7 8 , 4}$ & 84 & 89,6 \\
\hline $\mathbf{5 0}$ & 63 & $\mathbf{6 8 , 6}$ & $\mathbf{7 4 , 2}$ & $\mathbf{7 9 , 8}$ & 85,4 & 91,1 \\
\hline $\mathbf{6 0}$ & 64,4 & $\mathbf{7 0 , 1}$ & $\mathbf{7 5 , 7}$ & $\mathbf{8 1 , 4}$ & 87 & 92,6 \\
\hline $\mathbf{7 0}$ & 66 & $\mathbf{7 1 , 6}$ & $\mathbf{7 7 , 3}$ & $\mathbf{8 2 , 9}$ & 88,4 & 94,1 \\
\hline $\mathbf{8 0}$ & 67,4 & 73,1 & 78,7 & 84,4 & 90 & 95,6 \\
\hline $\mathbf{9 0}$ & 69 & 74,6 & 80,3 & 85,9 & 91,6 & 97,2 \\
\hline
\end{tabular}

\begin{tabular}{|c|c|c|c|c|c|c|}
\hline & 150 & 160 & 170 & 180 & 190 & 200 \\
\hline $\mathbf{2 0}$ & 6 & 6 & 6 & 7 & 8 & 8 \\
\hline $\mathbf{3 0}$ & 6 & 7 & 7 & 7 & 8 & 8 \\
\hline $\mathbf{4 0}$ & 6 & 7 & 7 & 7 & 8 & 8 \\
\hline $\mathbf{5 0}$ & 6 & 7 & 7 & 8 & 8 & 8 \\
\hline $\mathbf{6 0}$ & 6 & 7 & 7 & 8 & 8 & 8 \\
\hline $\mathbf{7 0}$ & 7 & 7 & 7 & 8 & 8 & 9 \\
\hline $\mathbf{8 0}$ & 7 & 7 & 7 & 8 & 8 & 9 \\
\hline $\mathbf{9 0}$ & 7 & 7 & 8 & 8 & 8 & 9 \\
\hline
\end{tabular}

(1) Z-score entre 2 et 4 : myélinopathie diffuse peu probable

(2) Z-score entre 4 et $7:$ myélinopathie diffuse possible

(3) Z-score $\geq 7$ : myélinopathie diffuse probable (Tankisi et al, 2005)

(4) Z-Score $\geq 8:$ myélinopathie diffuse définie 


\section{Latence F : 120\% LSN (n. tibial)}

\begin{tabular}{|c|c|c|c|c|c|c|c|c|c|c|c|c|c|}
\hline & 150 & 160 & 170 & 180 & 190 & 200 & & 150 & 160 & 170 & 180 & 190 & 200 \\
\hline 20 & 58,4 & 64,1 & 69,7 & 75,4 & 80,9 & 86,5 & 20 & 33 & 32 & 31 & 30 & 29 & 29 \\
\hline 30 & 59,9 & 65,5 & 71,2 & 76,8 & 82,4 & 88,1 & 30 & 33 & 32 & 31 & 30 & 29 & 28 \\
\hline 40 & 61,4 & 67,1 & 72,7 & 78,4 & 84 & 89,6 & 40 & 32 & 31 & 30 & 30 & 29 & 28 \\
\hline 50 & 63 & 68,6 & 74,2 & 79,8 & 85,4 & 91,1 & 50 & 32 & 31 & 30 & 29 & 29 & 28 \\
\hline 60 & 64,4 & 70,1 & 75,7 & 81,4 & 87 & 92,6 & 60 & 32 & 31 & 30 & 29 & 29 & 28 \\
\hline 70 & 66 & 71,6 & 77,3 & 82,9 & 88,4 & 94,1 & 70 & 31 & 30 & 30 & 29 & 28 & 28 \\
\hline 80 & 67,4 & 73,1 & 78,7 & 84,4 & 90 & 95,6 & 80 & 31 & 30 & 29 & 29 & 28 & 28 \\
\hline 90 & 69 & 74,6 & 80,3 & 85,9 & 91,6 & 97,2 & 90 & 31 & 30 & 29 & 29 & 28 & 28 \\
\hline \multicolumn{14}{|c|}{ (1) $\leq 20 \%$ : myélinopathie diffuse peu probable } \\
\hline \multicolumn{14}{|c|}{ (2) $\underline{e}$} \\
\hline \multicolumn{14}{|c|}{ (3) $\geq$} \\
\hline (4) & 4( & $m$ & ólin & atk & di & $d$ & & & & & & & \\
\hline
\end{tabular}




\section{Suivi longitudinal (n. médian) Lewis-Sumner}

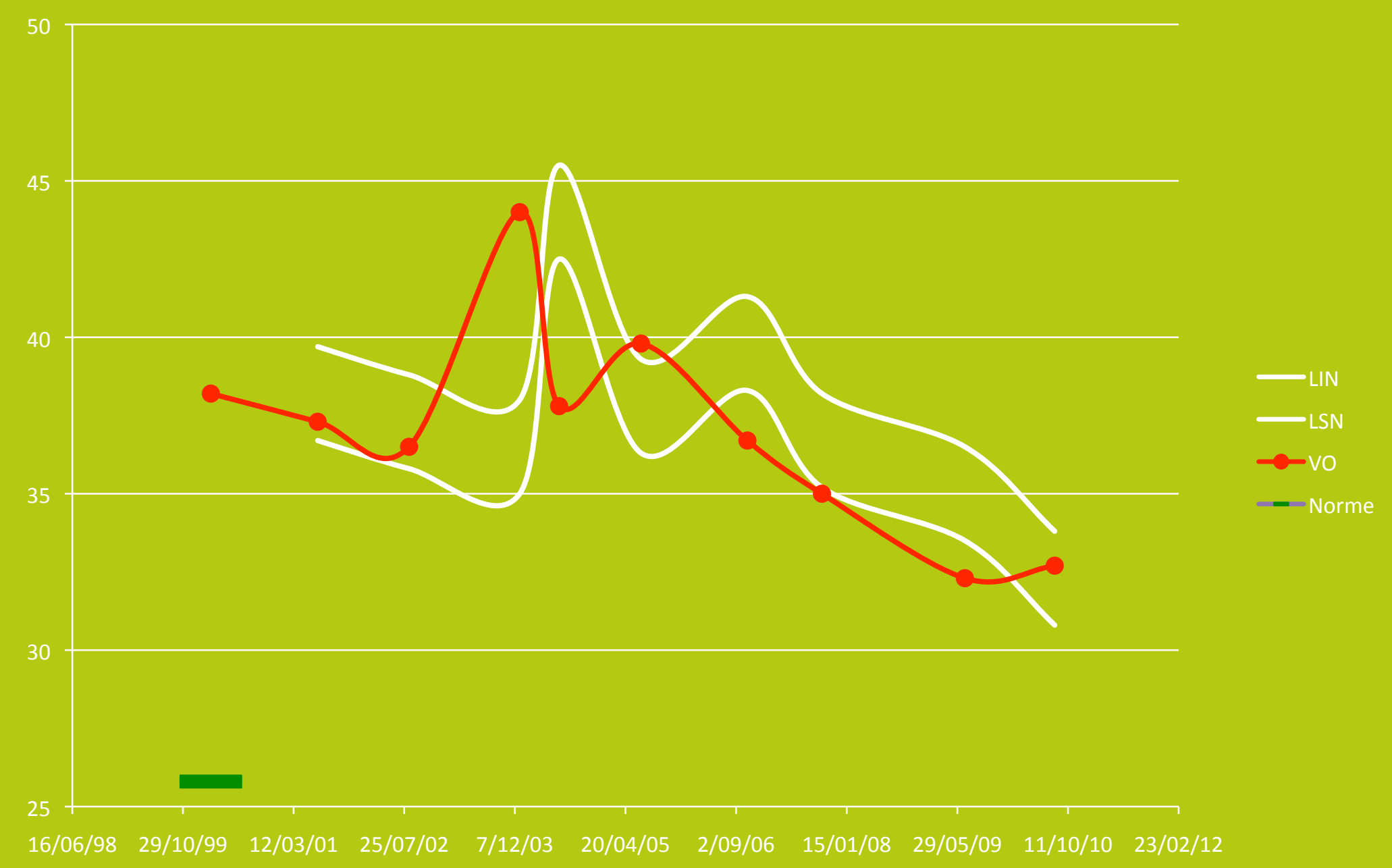




\section{Sclérose latérale amyotrophique}

(1) allongement modéré de la latence minimale

- perte des axones à conduction rapide (Cornblath et al, 1992)

- trouble du transport axonal (Felice, 1998)

- démyélinisation proximale (Bradley, 1987)

- hyperexcitabilité des grands $\mathrm{MN}$ avec

réactivation trop précoce du SI (Fisher, 1992)

(2) diminution de la persistance (Argyriou et al, 2006)

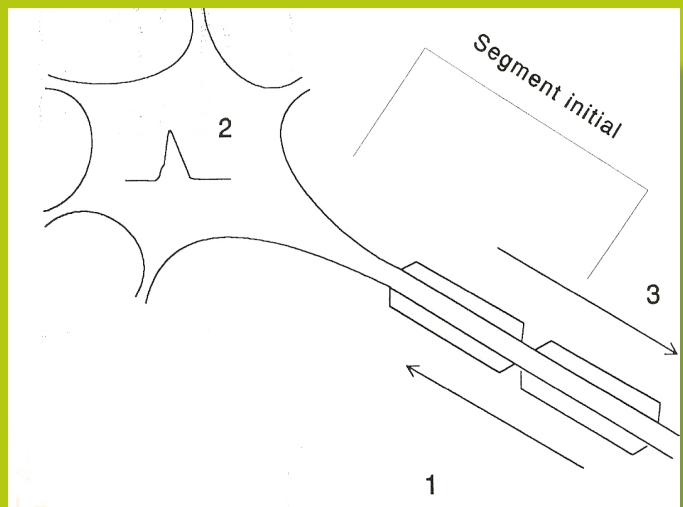

- hyperexcitabilité des $\mathrm{MN}$ avec réactivation trop précoce du SI

(Fisher, 1992 ; Peioglou-Harmoussi et al, 1987)

- perte motoneuronale 


\section{Sclérose latérale amyotrophique}

(1) augmentation de l’amplitude

- réinnervation collatérale (Eisen and Swash, 2001)

- augmentation du nombre de $\mathrm{MN}$ participant à la réponse $\mathrm{F}$

$>$ hyperexcitabilté motoneuronale (Argyriou et al, 2006)

(2) augmentation de la fréquence des ondes $\mathrm{F}$ monomorphes de grande amplitude (Petajan, 1985 ; Peioglou-Harmoussi et al, 1987 ;

Maisonobe, 2000)

- Fmax/Mmax $>15 \%$ : (Maisonobe, 2000) 
Motor Unit Number Estimation (MUNE) and Quantitative EMG

Editors: M.B. Bromberg rights reserved

Motor neurom disorders: novel electrophysiologic approach (MUUFDEC protocol)

F.C. Wang ${ }^{a, *}$, N. Le Forestier ${ }^{b}$, P. Gérard ${ }^{a}$, J.C. Willer ${ }^{b}$. V. Meininger ${ }^{b}$ D. Divea, A. Maertens de Noordhout ${ }^{a}$ and $P$. Bouche ${ }^{b}$

a Department of Neurophysiology, CHU Liège, 4000 Liège, Belgium

(1) Réponses $M$

- thénar X 2, TA X 2 : amp, surface

- score moteur, score/DD

(2) Décrément (SNR $3 \mathrm{~Hz}$ )

(3) Réponses $F$

- NI : M amp/(LDM X F persistance)

(de Carvalho et Swash, 2000)

- index $F$ : $F$ persistance $X F$ amp

(4) MUNE (thénar le - atteint)

- nb d'UM, nb d'UM/DD

- taille moyenne PUM

(5) $\%$ de changement $T 1 / T 2$

des $\neq$ paramètres

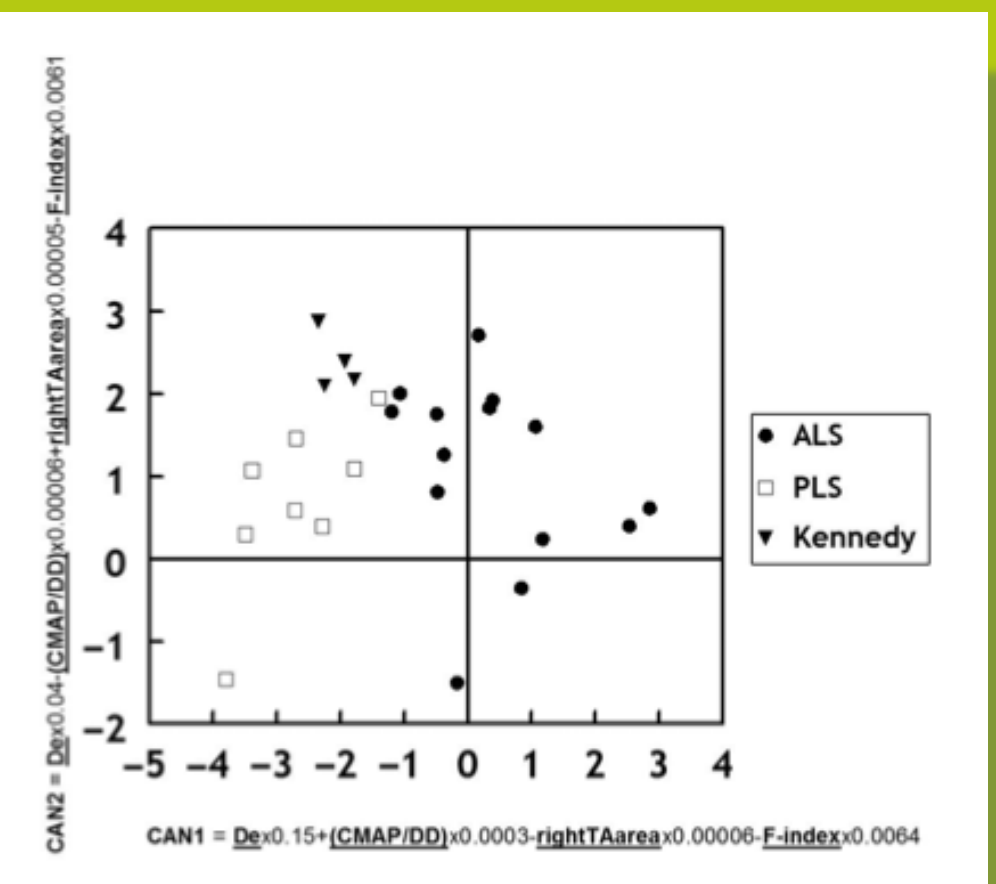




\section{$\mathrm{MERCl}$}

\section{Diaporama à télécharger >}

\section{Qsection liens du site http://cf-enmg.blogspot.com}

口ou directement sur le site http://enmgblog.blogspot.com 